\title{
Variational methods for fractional $q$-Sturm-Liouville problems
}

\section{Zeinab SI Mansour* (1)}

\section{"Correspondence:}

zsmansour@ksu.edu.sa

Present address: Department of

Mathematics, Faculty of Science,

King Saud University, Riyadh, Saudi

Arabia

Department of Mathematics,

Faculty of Science, Cairo University, Giza, Egypt

\section{Springer}

\begin{abstract}
In this paper, we formulate a regular q-fractional Sturm-Liouville problem (qFSLP) which includes the left-sided Riemann-Liouville and the right-sided Caputo $q$-fractional derivatives of the same order $\alpha, \alpha \in(0,1)$. We introduce the essential $q$-fractional variational analysis needed in proving the existence of a countable set of real eigenvalues and associated orthogonal eigenfunctions for the regular qFSLP when $\alpha>1 / 2$ associated with the boundary condition $y(0)=y(a)=0$. A criterion for the first eigenvalue is proved. Examples are included. These results are a generalization of the integer regular q-Sturm-Liouville problem introduced by Annaby and Mansour in (J. Phys. A, Math. Gen. 38:3775-3797, 2005; J. Phys. A, Math. Gen. 39:8747, 2006).
\end{abstract}

MSC: 39A13; 26A33; 49R05

Keywords: left- and right-sided Riemann-Liouville and Caputo q-derivatives; eigenvalues and eigenfunctions; $q$-fractional variational calculus

\section{Introduction}

In the joint paper of Sturm and Liouville [3], they studied the problem

$$
-\frac{d}{d x}\left(p \frac{d y}{d x}\right)+r(x) y(x)=\lambda w y(x), \quad x \in[a, b]
$$

with certain boundary conditions at $a$ and $b$. Here, the functions $p, w$ are positive on $[a, b]$ and $r$ is a real valued function on $[a, b]$. They proved the existence of non-zero solutions (eigenfunctions) only for special values of the parameter $\lambda$ which are called eigenvalues. For a comprehensive study of the contribution of Sturm and Liouville to the theory, see [4]. Recently, many mathematicians have become interested in a fractional version of (1.1), i.e., when the derivative is replaced by a fractional derivative like Riemann-Liouville derivative or Caputo derivative; see [5-10]. Iterative methods, variational method, and the fixed point theory are three different approaches used in proving the existence and uniqueness of solutions of Sturm-Liouville problems, $c f$. [4, 11, 12]. The calculus of variations has recently been developed to calculate the extremum of a functional that contains fractional derivatives, which is called the fractional calculus of variations; see for example [13-20]. In [5], Klimek et al. applied the methods of fractional variational calculus to prove the existence of a countable set of orthogonal solutions and corresponding eigenvalues. In $[1,2]$,

(c) 2016 Mansour. This article is distributed under the terms of the Creative Commons Attribution 4.0 International License (http://creativecommons.org/licenses/by/4.0/), which permits unrestricted use, distribution, and reproduction in any medium, provided you give appropriate credit to the original author(s) and the source, provide a link to the Creative Commons license, and indicate if changes were made. 
Annaby and Mansour introduced a $q$-version of (1.1), i.e., when the derivative is replaced by Jackson $q$-derivative. Their results are applied and developed in different respects; for example, see [21-26]. Throughout this paper $q$ is a positive number less than 1 . The set of non-negative integers is denoted by $\mathbb{N}_{0}$, and the set of positive integers is denoted by $\mathbb{N}$. For $t>0$,

$$
A_{q, t}:=\left\{t q^{n}: n \in \mathbb{N}_{0}\right\}, \quad A_{q, t}^{*}:=A_{q, t} \cup\{0\},
$$

and

$$
\mathcal{A}_{q, t}:=\left\{ \pm t q^{n}: n \in \mathbb{N}_{0}\right\}
$$

When $t=1$, we simply use $A_{q}, A_{q}^{*}$, and $\mathcal{A}_{q}$ to denote $A_{q, 1}, A_{q, 1}^{*}$, and $\mathcal{A}_{q, 1}$, respectively. In the following, we state the basic $q$-notations and notions we use in this article, $c f$. [27, 28].

For $n \in \mathbb{N}_{0}$, the $q$-shifted factorial $(a ; q)_{n}$ of $a \in \mathbb{C}$ is defined by

$$
(a ; q)_{0}:=1 \quad \text { and } \quad \text { for } n \in \mathbb{N}, \quad(a ; q)_{n}:=\prod_{k=1}^{n}\left(1-a q^{k-1}\right)
$$

The multiple $q$-shifted factorial for complex numbers $a_{1}, \ldots, a_{k}$ is defined by

$$
\left(a_{1}, a_{2}, \ldots, a_{k} ; q\right)_{n}:=\prod_{j=1}^{k}\left(a_{j} ; q\right)_{n} .
$$

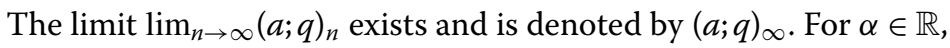

$$
(a ; q)_{\alpha}=\frac{(a ; q)_{\infty}}{\left(a q^{\alpha} ; q\right)_{\infty}}
$$

The $q$-gamma function, $[28,29]$, is defined for $z \in \mathbb{C}, z \neq-n, n \in \mathbb{N}$ by

$$
\Gamma_{q}(z):=\frac{(q ; q)_{\infty}}{\left(q^{z} ; q\right)_{\infty}}(1-q)^{1-z}, \quad 0<|q|<1
$$

Here we take the principal values of $q^{z}$ and $(1-q)^{1-z}$. Then $\Gamma_{q}(z)$ is a meromorphic function with poles at $z=-n, n \in \mathbb{N}$.

Let $\mu \in \mathbb{R}$ be fixed. A set $A \subseteq \mathbb{R}$ is called a $\mu$-geometric set if for $x \in A, \mu x \in A$. If $f$ is a function defined on a $q$-geometric set $A \subseteq \mathbb{R}$, the $q$-difference operator, $D_{q}$, is defined by

$$
D_{q} f(x):=\frac{f(x)-f(q x)}{x-q x}, \quad x \in A /\{0\}
$$

If $0 \in A$, we say that $f$ has $q$-derivative at zero if

$$
\lim _{n \rightarrow \infty} \frac{f\left(x q^{n}\right)-f(0)}{x q^{n}}, \quad x \in A,
$$

exists and does not depend on $x$. In this case, we shall denote this limit by $D_{q} f(0)$. In some literature the $q$-derivative at zero is defined to be $f^{\prime}(0)$ if it exists, $c f$. [30, 31], but the above 
definition is more suitable for our approach. The non-symmetric Leibniz rule

$$
D_{q}(f g)(x)=g(x) D_{q} f(x)+f(q x) D_{q} g(x)
$$

holds. Equation (1.7) can be symmetrized using the relation $f(q x)=f(x)-x(1-q) D_{q} f(x)$, giving the additional term $-x(1-q) D_{q} f(x) D_{q} g(x)$. The $q$-integration of Jackson [32] is defined for a function $f$ defined on a $q$-geometric set $A$ to be

$$
\int_{a}^{b} f(t) d_{q} t:=\int_{0}^{b} f(t) d_{q} t-\int_{0}^{a} f(t) d_{q} t, \quad a, b \in A,
$$

where

$$
\int_{0}^{x} f(t) d_{q} t:=\sum_{n=0}^{\infty} x q^{n}(1-q) f\left(x q^{n}\right), \quad x \in A,
$$

provided that the series converges. A function $f$ defined on $X$ is called $q$-regular at zero if

$$
\lim _{n \rightarrow \infty} f\left(x q^{n}\right)=f(0) \quad \text { for all } x \in X
$$

Let $C(X)$ denote the space of all $q$-regular at zero functions defined on $X$ with values in $\mathbb{R}$. $C(X)$ associated with the norm function

$$
\|f\|=\sup \left\{\left|f\left(x q^{n}\right)\right|: x \in X, n \in \mathbb{N}_{0}\right\},
$$

is a normed space. The $q$-integration by parts rule [33] is

$$
\int_{a}^{b} f(x) D_{q} g(x)=\left.f(x) g(x)\right|_{a} ^{b}+\int_{a}^{b} D_{q} f(x) g(q x) d_{q} x, \quad a, b \in X
$$

where $f, g$ are $q$-regular at zero functions.

For $p>0$, and $Y$ is $A_{q, t}$ or $A_{q, t}^{*}$, the space $L_{q}^{p}(Y)$ is the normed space of all functions defined on $Y$ such that

$$
\|f\|_{p}:=\left(\int_{0}^{t}|f(u)|^{p} d_{q} u\right)^{1 / p}<\infty
$$

If $p=2$, then $L_{q}^{2}(Y)$ associated with the inner product

$$
\langle f, g\rangle:=\int_{0}^{t} f(u) \overline{g(u)} d_{q} u
$$

is a Hilbert space. A weighted $L_{q}^{2}(Y, w)$ space is the space of all functions $f$ defined on $Y$, such that

$$
\int_{0}^{t}|f(u)|^{2} w(u) d_{q} u<\infty
$$


where $w$ is a positive function defined on $Y . L_{q}^{2}(Y, w)$ associated with the inner product

$$
\langle f, g\rangle:=\int_{0}^{t} f(u) \overline{g(u)} w(u) d_{q} u
$$

is a Hilbert space. The space of all $q$-absolutely functions on $A_{q, t}^{*}$ is denoted by $\mathcal{A} C_{q}\left(A_{q, t}^{*}\right)$ and defined as the space of all $q$-regular at zero functions $f$ satisfying

$$
\sum_{j=0}^{\infty}\left|f\left(u q^{j}\right)-f\left(u q^{j+1}\right)\right| \leq K \quad \text { for all } u \in A_{q, t}^{*}
$$

and $K$ is a constant depending on the function $f, c f$. [33], Definition 4.3.1. That is,

$$
\mathcal{A} C_{q}\left(A_{q, t}^{*}\right) \subseteq C_{q}\left(A_{q, t}^{*}\right)
$$

The space $\mathcal{A} C_{q}^{(n)}\left(A_{q, t}^{*}\right) \quad(n \in \mathbb{N})$ is the space of all functions defined on $X$ such that $f, D_{q} f, \ldots, D_{q}^{n-1} f$ are $q$-regular at zero and $D_{q}^{n-1} f \in \mathcal{A} C_{q}\left(A_{q, t}^{*}\right), c f$. [33], Definition 4.3.2. Also it has been proved in [33], Theorem 4.6, that a function $f \in \mathcal{A} C_{q}^{(n)}\left(A_{q, t}^{*}\right)$ if and only if there exists a function $\phi \in L_{q}^{1}\left(A_{q, t}^{*}\right)$ such that

$$
f(x)=\sum_{k=0}^{n-1} \frac{D_{q}^{k} f(0)}{\Gamma_{q}(k+1)} x^{k}+\frac{x^{n-1}}{\Gamma_{q}(n)} \int_{0}^{x}(q u / x ; q)_{n-1} \phi(u) d_{q} u, \quad x \in A_{q, t}^{*} .
$$

In particular, $f \in \mathcal{A} C\left(A_{q, t}^{*}\right)$ if and only if $f$ is $q$-regular at zero such that $D_{q} f \in L_{q}^{1}\left(A_{q, t}^{*}\right)$. It is worth noting that in [33], all the definitions and results we have just mentioned are defined and proved for functions defined on the interval [0,a] instead of $A_{q, t}^{*}$. In [34], Mansour studied the problem

$$
D_{q, a^{-}}^{\alpha} p(x)^{\mathrm{c}} D_{q, 0^{+}}^{\alpha} y(x)+\left(r(x)-\lambda w_{\alpha}(x)\right) y(x)=0, \quad x \in A_{q, a^{\prime}}^{*}
$$

where $p(x) \neq 0$ and $w_{\alpha}>0$ for all $x \in A_{q, a}^{*}, p, r, w_{\alpha}$ are real valued functions defined in $A_{q, a}^{*}$ and the associated boundary conditions are

$$
\begin{aligned}
& c_{1} y(0)+c_{2}\left[I_{q, a^{-}}^{1-\alpha} p^{\mathrm{c}} D_{q, 0^{+}}^{\alpha} y\right](0)=0, \\
& d_{1} y(a)+d_{2}\left[I_{q, a^{-}}^{1-\alpha} p^{\mathrm{c}} D_{q, 0^{+}}^{\alpha} y\right]\left(\frac{a}{q}\right)=0,
\end{aligned}
$$

with $c_{1}^{2}+c_{2}^{2} \neq 0$ and $d_{1}^{2}+d_{2}^{2} \neq 0$. it is proved that the eigenvalues are real and the eigenfunctions associated to different eigenvalues are orthogonal in the Hilbert space $L_{q}^{2}\left(A_{q, a}^{*}, w_{\alpha}\right)$. A sufficient condition on the parameter $\lambda$ to guarantee the existence and uniqueness of the solution is introduced by using the fixed point theorem, also a condition is imposed on the domain of the problem in order to prove the existence and uniqueness of solution for any $\lambda$. This paper is organized as follows. Section 2 is on the $q$-fractional operators and their properties which we need in the sequel. Cardoso [35] introduced basic Fourier series for functions defined on a $q$-linear grid of the form $\left\{ \pm q^{n}: n \in \mathbb{N}_{0}\right\} \cup\{0\}$. In Section 3, we reformulate Cardoso's results for functions defined on a $q$-linear grid of the 
form $\left\{ \pm a q^{n}: n \in \mathbb{N}_{0}\right\} \cup\{0\}$. In Section 4, we introduce a fractional $q$-analog for EulerLagrange equations for functionals defined in terms of Jackson $q$-integration and the integrand contains the left-sided Caputo fractional $q$-derivative. We also introduce a fractional $q$-isoperimetric problem. In Section 5, we use the variational $q$-calculus developed in Section 4 to prove the existence of a countable number of eigenvalues and orthogonal eigenfunctions for the fractional $q$-Sturm-Liouville problem with the boundary condition $y(0)=y(a)=0$. We also define the Rayleigh quotient and prove a criterion for the smallest eigenvalue.

\section{Fractional $q$-calculus}

This section includes the definitions and properties of the left-sided and right-sided Riemann-Liouville $q$-fractional operators which we need in our investigations.

The left-sided Riemann-Liouville $q$-fractional operator is defined by

$$
I_{q, a^{+}}^{\alpha} f(x)=\frac{x^{\alpha-1}}{\Gamma_{q}(\alpha)} \int_{a}^{x}(q t / x ; q)_{\alpha-1} f(t) d_{q} t
$$

This definition was introduced by Agarwal in [36] when $a=0$ and by Rajković et al. [37] for $a \neq 0$. The right-sided Riemann-Liouville $q$-fractional operator is defined by

$$
I_{q, b}^{\alpha} f(x)=\frac{1}{\Gamma_{q}(\alpha)} \int_{q x}^{b} t^{\alpha-1}(q x / t ; q)_{\alpha-1} f(t) d_{q} t
$$

see [34]. The left-sided Riemann-Liouville $q$-fractional operator satisfies the semigroup property

$$
I_{q, a^{+}}^{\alpha} I_{q, a^{+}}^{\beta} f(x)=I_{q, a^{+}}^{\alpha+\beta} f(x) .
$$

The case $a=0$ is proved in [36], while the case $a>0$ is proved in [37].

The right-sided Riemann-Liouville $q$-fractional operator satisfies the semigroup property [34]

$$
I_{q, b^{-}}^{\alpha} I_{q, b^{-}}^{\beta} f(x)=I_{q, b^{-}}^{\alpha+\beta} f(x), \quad x \in A_{q, b}^{*}
$$

for any function defined on $A_{q, b}$ and for any values of $\alpha$ and $\beta$.

For $\alpha>0$ and $\ulcorner\alpha\urcorner=m$, the left- and right-sided Riemann-Liouville fractional $q$-derivatives of order $\alpha$ are defined by

$$
D_{q, a^{+}}^{\alpha} f(x):=D_{q}^{m} I_{q, a^{+}}^{m-\alpha} f(x), \quad D_{q, b^{-}}^{\alpha} f(x):=\left(\frac{-1}{q}\right)^{m} D_{q^{-1}}^{m} I_{q, b^{-}}^{m-\alpha} f(x),
$$

the left- and right-sided Caputo fractional $q$-derivatives of order $\alpha$ are defined by

$$
{ }^{\mathrm{c}} D_{q, a^{+}}^{\alpha} f(x):=I_{q, a^{+}}^{m-\alpha} D_{q}^{m} f(x), \quad{ }^{\mathrm{c}} D_{q, b^{-}}^{\alpha}:=\left(\frac{-1}{q}\right)^{m} I_{q, b^{-}}^{m-\alpha} D_{q^{-1}}^{m} f(x) ;
$$

see [34]. From now on, we shall consider left-sided Riemann-Liouville and Caputo fractional $q$-derivatives when the lower point $a=0$ and right-sided Riemann-Liouville and 
Caputo fractional $q$-derivatives when $b=a$. According to [33],pp.124, $148, D_{q, 0^{+}}^{\alpha} f(x)$ exists if

$$
f \in L_{q}^{1}\left(A_{q, a}^{*}\right) \quad \text { such that } I_{q, 0^{+}}^{m-\alpha} f \in \mathcal{A} C_{q}^{(m)}\left(A_{q, a}^{*}\right)
$$

and ${ }^{\mathrm{c}} D_{q, a^{+}}^{\alpha} f$ exists if

$$
f \in \mathcal{A} C_{q}^{(m)}\left(A_{q, a}^{*}\right)
$$

The following proposition was proved in [34] but we add the proof here for convenience of the reader.

Proposition 2.1 Let $\alpha \in(0,1)$.

(i) Iff $\in L_{q}^{1}\left(A_{q, a}^{*}\right)$ such that $I_{q, 0^{+}}^{\alpha} f \in \mathcal{A} C_{q}\left(A_{q, a}^{*}\right)$ then

$$
{ }^{\mathrm{c}} D_{q, 0^{+}}^{\alpha} I_{q, 0^{+}}^{\alpha} f(x)=f(x)-\frac{I_{q, 0^{+}}^{\alpha} f(0)}{\Gamma_{q}(1-\alpha)} x^{-\alpha}
$$

Moreover, iff is bounded on $A_{q, a}^{*}$ then

$$
{ }^{\mathrm{c}} D_{q, 0^{+}}^{\alpha} I_{q, 0^{+}}^{\alpha} f(x)=f(x) .
$$

(ii) For any function $f$ defined on $A_{q, a}^{*}$,

$$
{ }^{\mathrm{c}} D_{q, a^{-}}^{\alpha} I_{q, a^{-}}^{\alpha} f(x)=f(x)-\frac{a^{-\alpha}}{\Gamma_{q}(1-\alpha)}(q x / a ; q)_{-\alpha}\left(I_{q, a^{-}}^{\alpha} f\right)\left(\frac{a}{q}\right)
$$

(iii) Iff $\in L_{q}^{1}\left(A_{q, a}\right)$ then

$$
D_{q, 0^{+}}^{\alpha} I_{q, 0^{+}}^{\alpha} f(x)=f(x) .
$$

(iv) For any function $f$ defined on $A_{q, a}^{*}$,

$$
D_{q, a^{-}}^{\alpha} I_{q, a^{-}}^{\alpha} f(x)=f(x)
$$

(v) Iff $\in \mathcal{A} C_{q}\left(A_{q, a}^{*}\right)$ then

$$
I_{q, 0^{+}}^{\alpha}{ }^{\mathrm{c}} D_{q, 0^{+}}^{\alpha} f(x)=f(x)-f(0) .
$$

(vi) Iff is a function defined on $A_{q, a}^{*}$ then

$$
I_{q, a^{-}}^{\alpha} D_{q, a^{-}}^{\alpha} f(x)=f(x)-\frac{a^{\alpha-1}}{\Gamma_{q}(\alpha)}(q x / a ; q)_{\alpha-1}\left(I_{q, a^{-}}^{1-\alpha} f\right)\left(\frac{a}{q}\right) .
$$

(vii) Iff is defined on $[0, a]$ such that $D_{q} f$ is continuous on $[0, a]$ then

$$
{ }^{\mathrm{c}} D_{q, 0^{+}}^{\alpha} f(x)=D_{q, 0^{+}}^{\alpha}[f(x)-f(0)]
$$


Proof The proof of (2.4) is a special case of [33], Eq. (5.7), but note that there is a misprint in Eq. (5.7); the summation should start from $i=1$. If $f$ is bounded on $A_{q, a}$, then $I_{q, 0^{+}}^{1-\alpha} f(0)=$ 0 , and (2.5) follows at once from (2.4). Now we prove (2.6). We have

$$
{ }^{\mathrm{c}} D_{q, a^{-}}^{\alpha} I_{q, a^{-}}^{\alpha} f(x)=\frac{1}{\Gamma_{q}(1-\alpha)} \int_{q x}^{a} t^{-\alpha}(q x / t ; q)_{-\alpha} D_{q}\left(I_{q, a^{-}}^{\alpha} f\right)\left(\frac{t}{q}\right) d_{q} t,
$$

where we used $-\frac{1}{q} D_{q^{-1}} f(x)=D_{q, x} f\left(\frac{x}{q}\right)$. Then applying the $q$-integration by parts formula (1.10) and using

$$
D_{q, t} t^{\beta}(q x / t ; q)_{\beta}=-[\beta] t^{\beta-1}(q x / t ; q)_{\beta-1}, \quad \beta \in \mathbb{R},[\beta]:=\frac{1-q^{\beta}}{1-q},
$$

we obtain

$$
{ }^{\mathrm{c}} D_{q, a^{-}}^{\alpha} I_{q, a^{-}}^{\alpha} f(x)=\frac{a^{-\alpha}}{\Gamma_{q}(1-\alpha)}(q x / a ; q)_{-\alpha}\left(I_{q, a^{-}}^{1-\alpha} f\right)\left(\frac{a}{q}\right)-I_{q, a^{-}}^{-\alpha} I_{q, a^{-}}^{\alpha} f(x) .
$$

Hence, the result follows from the semigroup property (2.3). Equation (2.7) was proved in [33], Eq. (4.66). The proof of (2.8) follows from the fact that

$$
D_{q, a^{-}}^{\alpha} I_{q, a^{-}}^{\alpha} f(x)=-\frac{1}{q} I_{q, a^{-}}^{1-\alpha} I_{q, a^{-}}^{\alpha} f(x)=-\frac{1}{q} D_{q^{-1}} I_{q, a^{-}} f(x)=f(x),
$$

where we used the semigroup property (2.3). The proof of (2.9) is a special case of [33], Eq. (5.6). The proof of (2.10) is similar to the proof of (2.8) and is omitted. Finally, the proof of (2.11) is a special case of [33], Eq. (5.8).

Set $X=A_{q, a}$ or $A_{q, a}^{*}$. Then

$$
C(X) \subseteq L_{q}^{2}(X) \subseteq L_{q}^{1}(X)
$$

Moreover, if $f \in C(X)$ then

$$
\|f\|_{1} \leq \sqrt{a}\|f\|_{2} \leq a\|f\| .
$$

We also have the following inequalities:

1. If $f \in C\left(A_{q, a}^{*}\right)$ then $I_{q, 0^{+}}^{\alpha} f \in C\left(A_{q, a}^{*}\right)$ and

$$
\left\|I_{q, 0^{+}}^{\alpha} f\right\| \leq \frac{a^{\alpha}}{\Gamma_{q}(\alpha+1)}\|f\|
$$

2. If $f \in L_{q}^{1}(X)$ then $I_{q, 0^{+}}^{\alpha} f \in L_{q}^{1}(X)$ and

$$
\left\|I_{q, 0^{+}}^{\alpha} f\right\|_{1} \leq M_{\alpha, 1}\|f\|_{1}, \quad M_{\alpha, 1}:=\frac{a^{\alpha}(1-q)^{\alpha}}{\left(1-q^{\alpha}\right)(q ; q)_{\infty}}
$$

3. If $f \in L_{q}^{2}(X)$ then $I_{q, 0^{+}}^{\alpha} f \in L_{q}^{2}(X)$ and

$$
\left\|I_{q, 0^{+}}^{\alpha} f\right\|_{2} \leq M_{\alpha, 2}\|f\|_{2},
$$


where

$$
M_{\alpha, 2}:=\frac{a^{\alpha}}{\Gamma_{q}(\alpha)} \sqrt{\frac{(1-q)}{\left(1-q^{2 \alpha}\right)}}\left(\int_{0}^{1}(q \xi ; q)_{\alpha-1}^{2} d_{q} \xi\right)^{1 / 2} .
$$

4. If $\alpha>\frac{1}{2}$ and $f \in L_{q}^{2}(X)$ then $I_{q, 0^{+}}^{\alpha} f \in C(X)$ and

$$
\left\|I_{q, 0^{+}}^{\alpha} f\right\| \leq \tilde{M}_{\alpha}\|f\|, \quad \tilde{M}_{\alpha}:=\frac{a^{\alpha-\frac{1}{2}}}{\Gamma_{q}(\alpha)}\left(\int_{0}^{1}(q \xi ; q)_{\alpha-1}^{2} d_{q} \xi\right)^{1 / 2} .
$$

5. Since $\|f\|_{2} \leq \sqrt{a}\|f\|$, we conclude that if $f \in C(X)$ then $I_{q, 0^{+}}^{\alpha} f \in L_{q}^{2}(X)$ and

$$
\left\|I_{q, 0^{+}}^{\alpha}\right\|_{2} \leq K_{\alpha}\|f\|, \quad K_{\alpha}:=\sqrt{a} M_{\alpha, 2}
$$

6. If $f \in C\left(A_{q, a}^{*}\right)$ then $I_{q, a}^{\alpha} f \in C\left(A_{q, a}^{*}\right)$ and

$$
\left\|I_{q, a^{-}}^{\alpha} f\right\| \leq c_{\alpha, 0}\|f\|, \quad c_{\alpha, 0}:=\frac{a^{\alpha}(1-q)^{\alpha}}{\left(1-q^{\alpha}\right)(q ; q)_{\infty}}
$$

7. If $f \in L_{q}^{1}(X)$ then $I_{q, a}^{\alpha} f \in L_{q}^{1}(X)$ and

$$
\left\|I_{q, a^{-}}^{\alpha} f\right\|_{1} \leq \begin{cases}\frac{(1-q)^{\alpha} a^{\alpha}}{\left(1-q^{\alpha}\right)(q ; q)_{\infty}}\|f\|_{1}, & \text { if } \alpha<1 \\ \frac{(1-q)^{\alpha-1} a^{\alpha-1}}{(q ; q)_{\infty}}\|f\|_{1}, & \text { if } \alpha \geq 1 .\end{cases}
$$

8. If $\alpha \neq \frac{1}{2}$ and $f \in L_{q}^{2}(X)$ then $I_{q, a^{-}}^{\alpha} f \in L_{q}^{1}(X)$ and

$$
\left\|I_{q, a}^{\alpha}-f\right\|_{2} \leq \begin{cases}\frac{(1-q)^{\alpha-\frac{1}{2}} a^{\alpha}}{\sqrt{1-q^{2 \alpha-1}}(q ; q)_{\infty}}\|f\|_{2}, & \text { if } \alpha<\frac{1}{2} \\ \frac{(1-q)^{\alpha} a^{\alpha}}{(q ; q)_{\infty} \sqrt{\left(1-q^{2 \alpha-1}\right)\left(1-q^{2 \alpha}\right)}}\|f\|_{2}, & \text { if } \alpha>\frac{1}{2} .\end{cases}
$$

The following lemmas are needed in the remaining sections.

Lemma 2.2 Let $\alpha>0$. If

(a) $f \in L_{q}^{1}(X)$ and $g$ is a bounded function on $A_{q, a}$, or

(b) $\alpha \neq \frac{1}{2}$ and $f$, $g$ are $L_{q}^{2}(X)$ functions,

then

$$
\int_{0}^{a} g(x) I_{q, 0^{+}}^{\alpha} f(x) d_{q^{x}} x=\int_{0}^{a} f(x) I_{q, a^{-}}^{\alpha} g(x) d_{q^{x}}
$$

Proof The condition (a) or (b) of the present lemma ensures the convergence of the $q$-integrals in (2.17). Since

$$
\int_{0}^{a} g(x) I_{q, 0+}^{\alpha} f(x) d_{q} x=\frac{1}{\Gamma_{q}(\alpha)} \int_{0}^{a} g(x) x^{\alpha-1} \int_{0}^{x}(q t / x ; q)_{\alpha-1} f(t) d_{q} t d_{q} x,
$$


from the conditions on the functions $f$ and $g$, the double $q$-integral is absolutely convergent, therefore we can interchange the order of the $q$-integrations to obtain

$$
\begin{aligned}
\int_{0}^{a} g(x) I_{a+}^{\alpha} f(x) d_{q} x & =\int_{0}^{a} f(t) \frac{1}{\Gamma_{q}(\alpha)} \int_{q t}^{a} x^{\alpha-1}(q t / x ; q)_{\alpha-1} g(x) d_{q^{\prime}} x d_{q} t \\
& =\int_{0}^{a} f(t) I_{q, a^{-}}^{\alpha} g(t) d_{q} t .
\end{aligned}
$$

Lemma 2.3 Let $\alpha \in(0,1)$.

(a) If $g \in L_{q}^{1}\left(A_{q, a}^{*}\right)$ such that $I_{q}^{1-\alpha} g \in \mathcal{A} C_{q}\left(A_{q, a}^{*}\right)$, and $D_{q}^{i} f \in C\left(A_{q, a}^{*}\right)(i=0,1)$ then

$$
\int_{0}^{a} f(x) D_{q, 0^{+}}^{\alpha} g(x) d_{q^{\prime}} x=-\left.f\left(\frac{x}{q}\right) I_{q, 0^{+}}^{1-\alpha} g(x)\right|_{x=0} ^{a}+\int_{0}^{a} g(x)^{\mathrm{c}} D_{q, a}^{\alpha} f(x) d_{q} x .
$$

(b) If $f \in \mathcal{A} C_{q}\left(A_{q, a}^{*}\right)$, and $g$ is a bounded function on $A_{q, a}^{*}$ such that $D_{q, a^{-}}^{\alpha} g \in L_{q}^{1}\left(A_{q, a}^{*}\right)$ then

$$
\int_{0}^{a} g(x)^{\mathrm{c}} D_{q, 0^{+}}^{\alpha} f(x) d_{q^{\prime}} x=\left.\left(I_{q, a^{-}}^{1-\alpha} g\right)\left(\frac{x}{q}\right) f(x)\right|_{x=0} ^{a}+\int_{0}^{a} f(x) D_{q, a^{-}}^{\alpha} g(x) d_{q} x
$$

Proof The conditions on the functions $f$ and $g$ guarantee the convergence of the $q$-integrals in (2.18) and (2.19), and their proofs follow from Lemma 2.2 and the $q$-integration by parts rule (1.10).

\section{Basic Fourier series on $q$-linear grid and some properties}

The purpose of this section is to reformulate Cardoso's results of Fourier series expansions for functions defined on the $q$-linear grid $\mathcal{A}_{q}:=\left\{q^{n}, n \in \mathbb{N}_{0}\right\}$ to functions defined on $q$ linear grids $\mathcal{A}_{q, a}:=\left\{ \pm a q^{n}, n \in \mathbb{N}_{0}\right\}, a>0$.

Cardoso in [35] defined the space of all $q$-linear Hölder functions on the $q$-linear grid $\mathcal{A}_{q}$. We generalize his definition for functions defined on a $q$-linear grid of the form $\mathcal{A}_{q, a}$, $a>0$.

Definition 3.1 A function $f$ defined on $\mathcal{A}_{q, a}, a>0$, is called a $q$-linear Hölder of order $\lambda$ if there exists a constant $M>0$ such that

$$
\left|f\left( \pm a q^{n-1}\right)-f\left( \pm a q^{n}\right)\right| \leq M q^{n \lambda} \text { for all } n \in \mathbb{N} \text {. }
$$

Definition 3.2 The $q$-trigonometric functions $S_{q}(z)$ and $C_{q}(z)$ are defined for $z \in \mathbb{C}$ by $[35,38]$

$$
\begin{aligned}
& S_{q}(z)=\sum_{n=0}^{\infty}(-1)^{n} \frac{q^{n\left(n+\frac{1}{2}\right)} z^{2 n+1}}{(q ; q)_{2 n+1}}=\frac{z}{1-q} \phi_{1}\left(0 ; q^{3} ; q^{2}, q^{3 / 2} z^{2}\right), \\
& C_{q}(z)=\sum_{n=0}^{\infty}(-1)^{n} \frac{q^{n\left(n-\frac{1}{2}\right)} z^{2 n}}{(q ; q)_{2 n}}={ }_{1} \phi_{1}\left(0 ; q ; q^{2}, q^{1 / 2} z^{2}\right) .
\end{aligned}
$$

One can verify that

$$
D_{q, z} S_{q}(w z)=\frac{w}{1-q} C_{q}(\sqrt{q} w z)
$$




$$
D_{q, z} C_{q}(w z)=-\frac{w}{1-q} S_{q}(\sqrt{q} w z)
$$

where $z \in \mathbb{C}$ and $w \in \mathbb{C}$ is a fixed parameter. A modification of the orthogonality relation given in [38], Theorem 4.1, is the following.

Theorem 3.3 Let $w$ and $w^{\prime}$ be roots of $S_{q}(z)$, and $\mu(w):=(1-q) C_{q}\left(q^{1 / 2} w\right) S_{q}^{\prime}(w)$. Then

$$
\begin{aligned}
& \int_{-a}^{a} C_{q}\left(\frac{q^{\frac{1}{2}} w x}{a}\right) C_{q}\left(\frac{q^{\frac{1}{2}} w^{\prime} x}{a}\right) d_{q} x= \begin{cases}0, & \text { if } w \neq w^{\prime}, \\
2 a, & \text { if } w=w^{\prime}=0, \\
a \mu(w), & \text { if } w=w^{\prime} \neq 0,\end{cases} \\
& \int_{-a}^{a} S_{q}\left(\frac{q w x}{a}\right) S_{q}\left(\frac{q w^{\prime} x}{a}\right) d_{q} x= \begin{cases}0, & \text { if } w \neq w^{\prime}, \\
a q^{-1 / 2} \mu(w), & \text { if } w=w^{\prime} .\end{cases}
\end{aligned}
$$

Cardoso introduced a sufficient condition for the uniform convergence of the basic Fourier series

$$
S_{q}(f):=\frac{a_{0}}{2}+\sum_{k=1}^{\infty} a_{k} C_{q}\left(q^{1 / 2} w_{k} x\right)+b_{k} S_{q}\left(q w_{k} x\right),
$$

where $a_{0}=\int_{-1}^{1} f(t) d_{q} t$ and for $k=1,2, \ldots$,

$$
\begin{gathered}
a_{k}=\frac{1}{\mu_{k}} \int_{-1}^{1} f(t) C_{q}\left(q^{1 / 2} w_{k} t\right) d_{q} t, \quad b_{k}=\frac{1}{\mu_{k}} \int_{-1}^{1} f(t) S_{q}\left(q w_{k} t\right) d_{q} t, \\
\mu_{k}=(1-q) C_{q}\left(q^{1 / 2} w_{k}\right) S_{q}^{\prime}\left(w_{k}\right)
\end{gathered}
$$

on the $q$-linear grid $\mathcal{A}_{q}$, where $\left\{w_{k}: k \in \mathbb{N}\right\}$ is the set of positive zeros of $S_{q}(z)$. Cardoso proved that $\mu_{k}=O\left(q^{-2 k^{2}}\right)$ as $k \rightarrow \infty$ for any $q \in(0,1)$. In the following we give a modified version of Cardoso's result for any function defined on the $q$-linear grid $\mathcal{A}_{q, a}, a>0$.

Theorem 3.4 If $\in C\left(\mathcal{A}_{q, a}^{*}\right)$ is a q-linear Hölder function of order $\lambda>\frac{1}{2}$, then the $q$-Fourier series

$$
S_{q}(f):=\frac{a_{0}}{2}+\sum_{k=1}^{\infty} a_{k} C_{q}\left(q^{1 / 2} \frac{w_{k} x}{a}\right)+b_{k} S_{q}\left(q \frac{w_{k} x}{a}\right),
$$

where $a_{0}=\frac{1}{a} \int_{-a}^{a} f(t) d_{q}$ tand, for $k=1,2, \ldots$,

$$
a_{k}(f)=\frac{1}{a \mu_{k}} \int_{-a}^{a} f(t) C_{q}\left(q^{1 / 2} \frac{w_{k} t}{a}\right) d_{q} t, \quad b_{k}(f)=\frac{\sqrt{q}}{a \mu_{k}} \int_{-a}^{a} f(t) S_{q}\left(q \frac{w_{k} t}{a}\right) d_{q} t,
$$

converges uniformly to the function $f$ on the q-linear grid $\mathcal{A}_{q, a}$.

Proof The proof is a modification of the proof of [35], Theorem 4.1, and is omitted.

Remark 3.5 We replaced the condition

$$
f\left(0^{+}\right)=f\left(0^{-}\right)
$$


where

$$
f\left(0^{+}\right):=\lim _{x \rightarrow 0^{+}} f(x), \quad f\left(0^{-}\right):=\lim _{x \rightarrow 0^{-}} f(x),
$$

in [35], Theorem 4.1, by the weakest condition that $f$ is $q$-regular at zero because (3.2) is only needed to guarantee that $\lim _{n \rightarrow \infty} f\left(q^{n-1 / 2}\right)=\lim _{n \rightarrow \infty} f\left(-q^{n-1 / 2}\right)$ and this holds if $f$ is $q$-regular at zero. See [33], Eq. (1.22), for a function which is $q$-regular at zero but not continuous at zero.

A modified version of [35], Theorem 3.5, is the following.

Theorem 3.6 If there exists $c>1$ such that

$$
\int_{-a}^{a} f(t) C_{q}\left(\sqrt{q} \frac{w_{k} t}{a}\right)=O\left(q^{c k}\right) \text { and } \int_{-a}^{a} f(t) S_{q}\left(q \frac{w_{k} t}{a}\right)=O\left(q^{c k}\right) \text { as } k \rightarrow \infty
$$

then the q-Fourier series (3.1) converges uniformly on $\mathcal{A}_{q, a}$.

A modified version of [35], Corollary 4.3, is the following.

Corollary 3.7 Iff is continuous and piecewise smooth on a neighborhood of the origin, then the corresponding $q$-Fourier series $S_{q}(f)$ converges uniformly to $f$ on the q-linear grid $\mathcal{A}_{q, a}$.

Theorem 3.8 Iff $\in C\left(\mathcal{A}_{q, a}^{*}\right)$ is a q-linear Hölder odd function of order $\lambda>\frac{1}{2}$ and satisfying $f(0)=f(a)=0$, then the q-Fourier series

$$
S_{q}(f):=\sum_{k=1}^{\infty} c_{k} S_{q}\left(\frac{w_{k} x}{a}\right)
$$

where

$$
c_{k}(f)=c_{k}=\frac{2}{a \sqrt{q} \mu_{k}} \int_{0}^{a} f(t) S_{q}\left(\frac{w_{k} t}{a}\right) d_{q} t,
$$

converges uniformly to the function $f$ on the q-linear grid $\mathcal{A}_{q, a}$.

Proof The proof follows from (3.4) by considering the function $g(x):=f(q x), x \in \mathcal{A}_{q, a}$. Since it is odd, we have $a_{k}=0$ for $k=0,1, \ldots$, and

$$
b_{k}(f)=\sqrt{q} \mu_{k} \int_{-a}^{a} g(t) S_{q}\left(\frac{q w_{k} t}{a}\right) d_{q} t
$$

making the substitution $u=q t$ and using the fact that $g$ is an odd function, we obtain the required result.

Definition 3.9 Let $\left(f_{n}\right)_{n}$ be a sequence of functions in $C\left(\mathcal{A}_{q, a}^{*}\right)$. We say that $f_{n}$ converges to a function $f$ in $q$-mean if

$$
\lim _{n \rightarrow \infty} \sqrt{\int_{-a}^{a}\left|f_{n}(x)-f(x)\right|^{2} d_{q} x}=0 .
$$


Proposition 3.10 If $g \in C\left(\mathcal{A}_{q, a}^{*}\right)$ is an odd function satisfying $D_{q}^{k} g(k=0,1,2)$ is a continuous and piecewise smooth function in a neighborhood of zero, satisfying the boundary condition

$$
g(0)=g(a)=0,
$$

then $g$ can be approximated in the q-mean by a linear combination

$$
g_{n}(x)=\sum_{r=0}^{n} c_{r}^{(n)} S_{q}\left(\frac{w_{r} x}{a}\right)
$$

where at the same time $D_{q}^{k} g_{n}(k=1,2)$ converges in $q$-mean to the $D_{q}^{k} g$. Moreover, the coefficients $c_{r}^{(n)}$ need not depend on $n$ and can be written simply as $c_{r}$.

Proof We consider the $q$-sine Fourier transform of $D_{q}^{2} g$. Hence

$$
D_{q}^{2} g(x)=\sum_{k=1}^{\infty} b_{k} S_{q}\left(\frac{q w_{k} x}{a}\right)=\lim _{n \rightarrow \infty} \gamma_{n}(x), \quad x \in A_{q, a}
$$

where

$$
\gamma_{n}(x)=\sum_{k=1}^{n} b_{k} S_{q}\left(\frac{q w_{k} x}{a}\right), \quad b_{k}=\frac{\sqrt{q}}{a \mu_{k}} \int_{0}^{a} D_{q}^{2} g(x) S_{q}\left(\frac{q w_{k} x}{a}\right) d_{q} x .
$$

Consequently,

$$
\lim _{n \rightarrow \infty} \int_{0}^{a}\left|D_{q}^{2} g(x)-\gamma_{n}(x)\right|^{2} d_{q} x=0 .
$$

Hence

$$
D_{q} g(x)-D_{q} g(0)=\int_{0}^{x} D_{q}^{2} g(x) d_{q} x=\frac{a(1-q)}{\sqrt{q}} \sum_{k=1}^{\infty} \frac{b_{k}}{w_{k}}\left(-C_{q}\left(\frac{q^{1 / 2} w_{k} x}{a}\right)+1\right) .
$$

Applying the $q$-integration by parts rule (1.10) gives

$$
a_{k}\left(D_{q} g\right)=-\frac{a(1-q)}{\sqrt{q} w_{k}} b_{k}\left(D_{q}^{2} g\right) .
$$

That is,

$$
D_{q} g(x)-D_{q} g(0)=\sum_{k=1}^{\infty} a_{k}\left(D_{q} g\right)\left(C_{q}\left(\frac{q^{1 / 2} w_{k} x}{a}\right)-1\right) .
$$

Hence

$$
D_{q} g(x)=\sum_{k=1}^{\infty} a_{k}\left(D_{q} g\right) C_{q}\left(\frac{q^{1 / 2} w_{k} x}{a}\right), \quad x \in A_{q, a}^{*} .
$$


Note that $a_{0}\left(D_{q} g\right)=0$ because $g(0)=g(a)=0$. Again by $q$-integrating the two sides of (3.5), we obtain

$$
g(x)=\sum_{k=1}^{\infty} a_{k}\left(D_{q} g\right) \frac{a(1-q)}{w_{k}} S_{q}\left(\frac{w_{k} x}{a}\right), \quad x \in A_{q, a}^{*} .
$$

One can verify that

$$
b_{k}(g)=\frac{a(1-q)}{w_{k}} a_{k}\left(D_{q} g\right) .
$$

Hence the right-hand sides of (3.5) and (3.6) are the $q$-Fourier series of $D_{q} g$ and $g$, respectively. Hence the convergence is uniform in $C\left(\mathcal{A}_{q, a}^{*}\right)$ and $L_{q^{2}}\left(\mathcal{A}_{q, a}^{*}\right)$ norms.

\section{$4 q$-Fractional variational problems}

The calculus of variations is as old as the calculus itself, and has many applications in physics and mechanics. As the calculus has two forms, the continuous calculus with the power concept of limits, and the discrete calculus which also is called the calculus of finite differences, the calculus of variations has also both the discrete and the continuous forms. For a brief history of the continuous calculus of variations, see [39]. The discrete calculus of variations started in 1948 by Fort in his book [40] where he devoted a chapter to the finite analog of the calculus of variations, and he introduced a necessary condition analog to the Euler equation and also a sufficient condition. The paper of Cadzow [41], 1969, was the first paper published in this field, then Logan developed the theory in his $\mathrm{PhD}$ thesis [42], 1970, and in a series of papers [43-46]. See also the PhD thesis of Harmsen [47] for a brief history for the discrete variational calculus; and for the developments in the theory, see [48-54]. In 2004, a $q$-version of the discrete variational calculus is introduced by Bangerezako in [55] for functions defined in the form

$$
J(y(x))=\int_{q^{\alpha}}^{q^{\beta}} x F\left(x, y(x), D_{q} y(x), \ldots, D_{q}^{k} y(x)\right) d_{q} x,
$$

where $q^{\alpha}$ and $q^{\beta}$ are in the uniform lattice $A_{q, a}^{*}$ for some $a>0$ such that $\alpha>\beta$, provided that the boundary conditions

$$
D_{q}^{j} y\left(q^{\alpha}\right)=D_{q}^{j} y\left(q^{\beta+1}\right)=c_{j} \quad(j=0,1, \ldots, k-1) .
$$

He introduced a $q$-analog of the Euler-Lagrange equation which he applied to solve certain isoperimetric problem. Then, in 2005, Bangerezako [56] introduced certain $q$-variational problems on a nonuniform lattice. In $[57,58]$, Malinowska, and Torres introduced the Hahn quantum variational calculus. They derived the Euler-Lagrange equation associated with the variational problem

$$
J(y)=\int_{a}^{b} F\left(t, y(q t+w), D_{q, w} y(t)\right) d_{q, w} t
$$


under the boundary condition $y(a)=\alpha, y(b)=\beta$ where $\alpha$ and $\beta$ are constants and $D_{q, w}$ is the Hahn difference operator defined by

$$
D_{q, w} f(t)= \begin{cases}\frac{f(q t+w)-f(t)}{(q+w)-t}, & \text { if } t \neq \frac{w}{1-q}, \\ f^{\prime}(0), & \text { if } t=\frac{w}{1-q} .\end{cases}
$$

Problems of the classical calculus of variations with integrand depending on fractional derivatives instead of ordinary derivatives are first introduced by Agrawal [17] in 2002. Then he extended his result for variational problems including Riesz fractional derivatives in [18]. Numerous works have been dedicated to the subject since Agrawal's work. See for example [5, 13-16, 59-61].

In this section, we shall derive Euler-Lagrange equation for a $q$-variational problem when the integrand includes a left-sided $q$-Caputo fractional derivative and we also solve a related isoperimetric problem. From now on, we fix $\alpha \in(0,1)$, and define a subspace of $C\left(A_{q, a}^{*}\right)$ by

$$
{ }_{0} E_{a}^{\alpha}=\left\{y \in \mathcal{A} C\left(A_{q, a}^{*}\right):{ }^{\mathrm{c}} D_{q, 0^{+}}^{\alpha} y \in C\left(A_{q, a}^{*}\right)\right\},
$$

and the space of variations ${ }^{c} \operatorname{Var}(0, a)$ for the Caputo $q$-derivative by

$$
{ }^{\mathrm{c}} \operatorname{Var}(0, a)=\left\{h \in{ }_{0} E_{a}^{\alpha}: h(0)=h(a)=0\right\} .
$$

For a function $f\left(x_{1}, x_{2}, \ldots, x_{n}\right)(n \in \mathbb{N})$ by $\partial_{i} f$ we mean the partial derivative of $f$ with respect to the $i$ th variable, $i=1,2, \ldots, n$. In the sequel, we shall need the following definition from [62].

Definition 4.1 Let $A \subseteq \mathbb{R}$ and $g: A \times]-\bar{\theta}, \bar{\theta}\left[\rightarrow \mathbb{R}\right.$. We say that $g(t, \cdot)$ is continuous at $\theta_{0}$ uniformly in $t$, if and only if $\forall \epsilon>0, \exists \delta>0$ such that

$$
\left|\theta-\theta_{0}\right|<\delta \longrightarrow\left|g(t, \theta)-g\left(t, \theta_{0}\right)\right|<\epsilon \quad \text { for all } t \in A .
$$

Furthermore, we say that $g(t, \cdot)$ is differentiable at $\theta_{0}$ uniformly in $t$ if and only if $\forall \epsilon>0$, $\exists \delta>0$ such that

$$
\left|\theta-\theta_{0}\right|<\delta \rightarrow\left|\frac{g(t, \theta)-g\left(t, \theta_{0}\right)}{\theta-\theta_{0}}-\delta_{2} g\left(t, \theta_{0}\right)\right|<\epsilon \quad \text { for all } t \in A \text {. }
$$

We now present first order necessary conditions of optimality for functionals, defined on $_{0} E_{a}^{\alpha}$, of the type

$$
J(y)=\int_{0}^{a} F\left(x, y,{ }^{\mathrm{c}} D_{q, 0^{+}}^{\alpha}\right) d_{q} x, \quad 0<\alpha<1,
$$

where $F: A_{q, a}^{*} \times \mathbb{R} \times \mathbb{R} \rightarrow \mathbb{R}$ is a given function. We assume that:

1. The functions $(u, v) \rightarrow F(t, u, v)$ and $(u, v) \rightarrow \partial_{i} F(t, u, v)(i=2,3)$ are continuous functions uniformly on $A_{q, a}$.

2. $F\left(\cdot, y(\cdot),{ }^{\mathrm{c}} D_{q, 0^{+}}^{\alpha}(\cdot)\right), \delta_{i} F\left(\cdot, y(\cdot),{ }^{\mathrm{c}} D_{q, 0^{+}}^{\alpha}(\cdot)\right)(i=2,3)$ are $q$-regular at zero. 
3. $\delta_{3} F$ has a right Riemann-Liouville fractional $q$-derivative of order $\alpha$ which is $q$-regular at zero.

Definition 4.2 Let $y_{0} \in{ }_{0} E_{a}^{\alpha}$. Then $J$ has a local maximum at $y_{0}$ if

$\exists \delta>0$ such that $J(y) \leq J\left(y_{0}\right) \quad$ for all $y \in{ }_{0} E_{a}^{\alpha}$ with $\left\|y-y_{0}\right\|<\delta$,

and $J$ has a local minimum at $y_{0}$ if

$\exists \delta>0 \quad$ such that $\quad J(y) \geq J\left(y_{0}\right) \quad$ for all $y \in S$ with $\left\|y-y_{0}\right\|<\delta$.

$J$ is said the have a local extremum at $y_{0}$ if it has either a local maximum or local minimum.

Lemma 4.3 Let $\gamma \in L_{q}^{2}\left(A_{q, a}^{*}\right)$.

(i) If

$$
\int_{0}^{a} \gamma(x) h(x) d_{q} x=0
$$

for every $h \in L_{q}^{2}\left(A_{q, a}\right)$ then

$$
\gamma(x) \equiv 0 \quad \text { on } A_{q, a} .
$$

(ii) If (4.2) holds only for all functions $h \in L_{q}^{2}\left(A_{q, a}^{*}\right)$ satisfying $h(a)=0$ then

$$
\gamma(x) \equiv 0 \quad \text { on } A_{q, q a} .
$$

Moreover, in the two cases, if $\gamma$ is q-regular at zero, then $\gamma(0)=0$.

Proof To prove (i), we fix $k \in \mathbb{N}_{0}$ and set $h_{k}(x)=\left\{\begin{array}{ll}1, & x=a q^{k}, \\ 0, & \text { otherwise. }\end{array}\right.$ Then $h_{k} \in L_{q}^{2}(0, a)$. Substituting in (4.2) yields

$$
a q^{k}(1-q) \gamma\left(a q^{k}\right)=0, \quad \forall k \in \mathbb{N}_{0} .
$$

Thus, $\gamma\left(a q^{k}\right)=0$ for all $k \in \mathbb{N}_{0}$. Clearly if $\gamma$ is $q$-regular at zero, then

$$
\gamma(0):=\lim _{k \rightarrow \infty} \gamma\left(a q^{k}\right)=0
$$

The proof of (ii) is similar and is omitted.

Lemma 4.4 If $\alpha \in C\left(A_{q, a}^{*}\right)$ and

$$
\int_{0}^{a} \alpha(x) D_{q} h(x) d_{q} x=0
$$

for any function $h$ satisfying

1. $h$ and $D_{q}$ h are $q$-regular at zero,

2. $h(0)=h(a)=0$,

then $\alpha(x)=$ c for all $x \in A_{q, a}^{*}$ where $c$ is a constant. 
Proof Let $c$ be the constant defined by the relation $c=\frac{1}{a} \int_{0}^{a} \alpha(x) d_{q} x$. Let

$$
h(x):=\int_{0}^{x}[\alpha(\xi)-c] d_{q} \xi, \quad x \in A_{q, a}^{*} .
$$

So, $h$ and $D_{q} h$ are $q$-regular at zero functions such that $h(0)=h(a)=0$. We have

$$
\int_{0}^{a}[\alpha(x)-c] D_{q} h(x) d_{q} x=\int_{0}^{a} \alpha(x) D_{q} h(x) d_{q} x+\left.[\alpha(x)-c] h(x)\right|_{x=0} ^{a}=0
$$

on the other hand,

$$
\int_{0}^{a} \alpha(x) D_{q} h(x) d_{q} x=\int_{0}^{a}[\alpha(x)-c]^{2} d_{q} x=0 .
$$

Therefore, $\alpha(x)=c$ for all $x \in A_{q, a}$. But $\alpha$ is $q$-regular at zero, hence $\alpha(0)=0$. This yields the required result.

Theorem 4.5 Let $y \in{ }^{\mathrm{c}} \operatorname{Var}(0, a)$ be a local extremum of J. Then $y$ satisfies the EulerLagrange equation

$$
\partial_{2} F(x)+D_{q, a^{-}}^{\alpha} \partial_{3} F(x)=0, \quad \forall x \in A_{q, q a}^{*}
$$

Proof Let $y$ be a local extremum of $J$ and let $\eta$ be arbitrary but fixed variation function of $y$. Define

$$
\Phi(\epsilon)=J(y+\epsilon \eta)
$$

Since $y$ is a local extremum for $J$, and $J(y)=\Phi(0)$, it follows that 0 is a local extremum for $\phi$. Hence $\phi^{\prime}(0)=0$. But

$$
0=\phi^{\prime}(0)=\lim _{\epsilon \rightarrow 0} \frac{d}{d \epsilon} \phi(y+\epsilon \eta)=\int_{0}^{a}\left(\partial_{2} F \eta+\partial_{3} F^{\mathrm{c}} D_{q, 0^{+}}^{\alpha} \eta\right) d_{q} x .
$$

Using (2.18), we obtain

$$
0=\int_{0}^{a}\left(\partial_{2} F+{ }^{c} D_{q, a^{-}}^{\alpha} \partial_{3} F\right) \eta d_{q} x+\left.I_{q, a^{-}}^{1-\alpha} \partial_{3} F(x) \eta(x)\right|_{x=0} ^{a}
$$

Since $\eta$ is a variation function, $\eta(0)=\eta(a)=0$, and we have

$$
\int_{0}^{a}\left(\partial_{2} F+D_{q, a^{-}}^{\alpha} \partial_{3} F\right) \eta d_{q} x=0
$$

for any $\eta \in{ }^{\mathrm{c}} \operatorname{Var}(0, a)$. Consequently, from Lemma 4.3, we obtain (4.5) and this completes the proof.

\subsection{A $q$-fractional isoperimetric problem}

In the following, we shall solve the $q$-fractional isoperimetric problem: Given a functional $J$ as in (4.1), find which functions minimize (or maximize) $J$, when subject to the boundary 
conditions

$$
y(0)=y_{0}, \quad y(a)=y_{a}
$$

and the $q$-integral constraint

$$
I(y)=\int_{0}^{a} G\left(x, y,{ }^{\mathrm{c}} D_{q, 0^{+}}^{\alpha} y\right) d_{q^{x}}=l,
$$

where $l$ is a fixed real number. Here, similarly to before:

1. The functions $(u, v) \rightarrow G(t, u, v)$ and $(u, v) \rightarrow \partial_{i} G(t, u, v)(i=2,3)$ are continuous functions uniformly on $A_{q, a}$.

2. $G\left(\cdot, y(\cdot),{ }^{\mathrm{c}} D_{q, 0^{+}}^{\alpha}(\cdot)\right), \delta_{i} G\left(\cdot, y(\cdot),{ }^{\mathrm{c}} D_{q, 0^{+}}^{\alpha}(\cdot)\right)(i=2,3)$ are $q$-regular at zero.

3. $\delta_{3} G$ has a right Riemann-Liouville fractional $q$-derivative of order $\alpha$ which is $q$-regular at zero.

A function $y \in E$ that satisfies (4.6) and (4.7) is called admissible.

Definition 4.6 An admissible function $y$ is an extremal for $I$ in (4.7) if it satisfies the equation

$$
\partial_{2} G(x)+D_{q, a^{-}}^{\alpha} \partial_{3} G(x)=0, \quad \forall x \in A_{q, q a}^{*} .
$$

Theorem 4.7 Let y be a local extremum for J given by (4.1), subject to the conditions (4.6) and (4.7). If $y$ is not an extremal of the function $I$, then there exists a constant $\lambda$ such that $y$ satisfies

$$
\partial_{2} H(x)+D_{q, a^{-}}^{\alpha} \partial_{3} H(x)=0, \quad \forall x \in A_{q, q a}^{*},
$$

where $H:=F-\lambda G$.

Proof Let $\eta_{1}, \eta_{2} \in{ }^{\mathrm{c}} \operatorname{Var}(0, a)$ be two functions, and let $\epsilon_{1}$ and $\epsilon_{2}$ be two real numbers, and consider the new function of two parameters

$$
\breve{y}=y+\epsilon_{1} \eta_{1}+\epsilon_{2} \eta_{2} .
$$

The reason why we consider two parameters is that we can choose one of them as a function of the other in order for $\breve{y}$ to satisfy the $q$-integral constraint (4.7). Let

$$
\breve{I}\left(\epsilon_{1}, \epsilon_{2}\right)=\int_{0}^{a} G\left(x, \breve{y},{ }^{\mathrm{c}} D_{q, 0^{+}}^{\alpha} \breve{y}\right) d_{q} x-l .
$$

It follows by the $q$-integration by parts rule (1.10) that

$$
\left.\frac{\partial \breve{I}}{\partial \epsilon_{2}}\right|_{(0,0)}=\int_{0}^{a}\left(\partial_{2} G(x)+D_{q, a^{-}}^{\alpha} \partial_{3} G(x)\right) \eta_{2} d_{q} x .
$$

Since $y$ is not an extremal of $I$, there exists a function $\eta_{2}$ satisfying the condition $\left.\frac{\partial \breve{I}}{\partial \epsilon_{2}}\right|_{(0,0)} \neq 0$. Hence, from the fact that $\breve{I}(0,0)=0$ and the implicit function theorem, there 
exists a $C^{1}$ function $\epsilon_{2}(\cdot)$, defined in some neighborhood of zero, such that

$$
\breve{I}\left(\epsilon_{1}, \epsilon_{2}\left(\epsilon_{1}\right)\right)=0 .
$$

Therefore, there exists a family of variations of type (4.10) satisfying the $q$-integral constraint. To prove the theorem, we define a new function $\breve{J}\left(\epsilon_{1}, \epsilon_{2}\right)=J(\breve{y})$. Since $(0,0)$ is a local extremum of $\breve{J}$ subject to the constraint $\breve{I}(0,0)=0$, and $\nabla \breve{I}(0,0) \neq(0,0)$, by the Lagrange multiplier rule, see [63], there exists a constant $\lambda$ for which the following holds:

$$
\nabla \breve{J}(0,0) \neq(0,0)-\lambda \breve{I}(0,0)=(0,0)
$$

Simple calculation shows that

$$
\left.\frac{\partial \breve{J}}{\partial \epsilon_{1}}\right|_{(0,0)}=\int_{0}^{a}\left(\partial_{2} F(x)+D_{q, a^{-}}^{\alpha} \partial_{3} F(x)\right) \eta_{1} d_{q^{x}}
$$

and

$$
\left.\frac{\partial \breve{I}}{\partial \epsilon_{1}}\right|_{(0,0)}=\int_{0}^{a}\left(\partial_{2} G(x)+D_{q, a^{-}}^{\alpha} \partial_{3} G(x)\right) \eta_{1} d_{q^{\prime}} x
$$

Consequently,

$$
\int_{0}^{a}\left[\partial_{2} F(x)+D_{q, a^{-}}^{\alpha} \partial_{3} F(x)-\lambda\left(\partial_{2} G(x)+D_{q, a^{-}}^{\alpha} \partial_{3} G(x)\right)\right] \eta_{1} d_{q} x .
$$

Since $\eta_{1}$ is arbitrary, from Lemma 4.3 , we obtain

$$
\partial_{2} F(x)+D_{q, a^{-}}^{\alpha} \partial_{3} F(x)-\lambda\left(\partial_{2} G(x)+D_{q, a^{-}}^{\alpha} \partial_{3} G(x)\right)=0
$$

for all $x \in A_{q, q a}^{*}$. This is equivalent to (4.9) and completes the proof.

The functions

$$
\begin{aligned}
& e_{\alpha, \beta}(z ; q):=\sum_{n=0}^{\infty} \frac{z^{n}}{\Gamma_{q}(\alpha n+1)} ; \quad\left|z(1-q)^{\alpha}\right|<1, \\
& E_{\alpha, \beta}(z ; q):=\sum_{n=0}^{\infty} q^{\frac{\alpha}{2} n(n-1)} \frac{z^{n}}{\Gamma_{q}(\alpha n+1)} ; \quad z \in \mathbb{C},
\end{aligned}
$$

are $q$-analogs of the Mittag-Leffler function

$$
E_{\alpha, \beta}(z)=\sum_{n=0}^{\infty} \frac{z^{n}}{\Gamma_{q}(\alpha n+1)}, \quad z \in \mathbb{C} ;
$$

see [33]. We have

$$
{ }^{\mathrm{c}} D_{q, 0^{+}}^{\alpha} e_{\alpha, 1}(z ; q):=e_{\alpha, 1}(z ; q) ; \quad{ }^{\mathrm{c}} D_{q, 0^{+}}^{\alpha} E_{\alpha, 1}(z ; q)=E_{\alpha, 1}(q z ; q) .
$$


Example 4.8 Consider the fractional $q$-isoperimetric problem:

$$
\begin{aligned}
& J(y)=\int_{0}^{a}\left({ }^{\mathrm{c}} D_{q, 0^{+}}^{\alpha} y(x)\right)^{2} d_{q} x, \\
& I(y)=\int_{0}^{a} e_{\alpha, 1}\left(x^{\alpha} ; q\right)^{\mathrm{c}} D_{q, 0^{+}}^{\alpha} y(x) d_{q} x=l, \\
& y(0)=1, \quad y(a)=e_{\alpha, 1}\left(a^{\alpha} ; q\right),
\end{aligned}
$$

where $0<a(1-q)<1$. Then

$$
H=\left({ }^{\mathrm{c}} D_{q, 0^{+}}^{\alpha} y\right)^{2}-\lambda e_{\alpha, 1}(x ; q)^{\mathrm{c}} D_{q, 0^{+}}^{\alpha} y
$$

and

$$
\partial_{2} H+D_{q, a^{-}}^{\alpha} \partial_{3} H=D_{q, a^{-}}^{\alpha}\left(2^{\mathrm{c}} D_{q, 0^{+}}^{\alpha} y(x)-\lambda e_{\alpha, 1}(x ; q)\right) .
$$

Therefore a solution of the problem is $\lambda=2$ and $y(x)=e_{\alpha, 1}\left(x^{\alpha} ; q\right)$. Similarly a solution of the problem

$$
\begin{aligned}
& J(y)=\int_{0}^{a}\left({ }^{\mathrm{c}} D_{q, 0^{+}}^{\alpha} y(x)\right)^{2} d_{q} x, \\
& I(y)=\int_{0}^{a} E_{\alpha, 1}\left((q x)^{\alpha} ; q\right)^{\mathrm{c}} D_{q, 0^{+}}^{\alpha} y(x) d_{q} x=l, \\
& y(0)=1, \quad y(a)=E_{\alpha, 1}\left(a^{\alpha} ; q\right),
\end{aligned}
$$

where $a>0$ is $y(x)=E_{\alpha, 1}\left(x^{\alpha} ; q\right)$.

\section{Existence of discrete spectrum for a fractional $q$-Sturm-Liouville problem}

In this section, we use the $q$-calculus of variations we developed in Section 4 to investigate the existence of solutions of the qFSLP

$$
D_{q, a^{-}}^{\alpha} p(x)^{\mathrm{c}} D_{q, 0^{+}}^{\alpha} y(x)+r(x) y(x)=\lambda w_{\alpha} y(x), \quad x \in A_{q, q a}^{*},
$$

under the boundary condition

$$
y(0)=y(a)=0 .
$$

The proof of the main result of this section depends on the Arzelà-Ascoli theorem [64], p.156. The setting of this theorem is a compact metric space $X$. Let $C(X)$ denote the space of all continuous functions on $X$ with values in $\mathbb{C}$ or $\mathbb{R} . C(X)$ is associated with the metric function

$$
d(f, g)=\max \{|f(x)-g(x)|: x \in X\} .
$$

Theorem 5.1 (Arzelà-Ascoli theorem) If a sequence $\left\{f_{n}\right\}_{n}$ in $C(X)$ is bounded and equicontinuous then it has a uniformly convergent subsequence. 
In our $q$-setting, we take $X=A_{q, a}^{*}$. Hence $f \in C\left(A_{q, a}^{*}\right)$ if and only if $f$ is $q$-regular at zero, i.e.,

$$
f(0):=\lim _{n \rightarrow \infty} f\left(a q^{n}\right)
$$

Remark 5.2 A question may be raised as to why in (5.1) we have only $x \in A_{q, q a}^{*}$ instead of $A_{q, a}^{*}$. The reason for that is that the qFSLP (5.1)-(5.2) will be solved by using the $q$-fractional isoperimetric problem developed in Theorem 4.7, and its $q$-Euler-Lagrange equation (4.9) holds only for $x \in A_{q, q a}^{*}$. Also, in order for (5.1) to hold at $x=a$, we should have $D_{q, a^{-}}^{\alpha}\left(p(\cdot)^{\mathrm{c}} D_{q, 0^{+}}^{\alpha} y(\cdot)\right)(a)=0$ and this holds only if $p(a)^{\mathrm{c}} D_{q, 0^{+}}^{\alpha} y(a)=0$, which may not hold.

Theorem 5.3 Let $\frac{1}{2}<\alpha<1$. Assume that the functions $p, r, w_{\alpha}$ are defined on $A_{q, a}^{*}$ and satisfying the conditions:

(i) $w_{\alpha}$ is a positive continuous function on $[0, a]$ such that $D_{q}^{k} \frac{1}{w_{\alpha}}(k=0,1,2)$ are bounded functions on $A_{q, a}$,

(ii) $r$ is a bounded function on $A_{q, a}$,

(iii) $p \in C\left(A_{q, a}^{*}\right)$ such that $\inf _{x \in A_{q, a}} p(x)>0$, and $\sup _{x \in A_{q, a}}\left|\frac{r(x)}{w_{\alpha}(x)}\right|<\infty$.

The q-fractional Sturm-Liouville problem (5.1)-(5.2) has an infinite number of eigenvalues $\lambda^{(1)}, \lambda^{(2)}, \ldots$, and to each eigenvalue $\lambda^{(n)}$ there is a corresponding eigenfunction $y^{(n)}$, which is unique up to a constant factor. Furthermore, the eigenfunctions $y^{(n)}$ form an orthogonal set of solutions in the Hilbert space $L_{q}^{2}\left(A_{q, a}^{*}, w_{\alpha}\right)$.

Proof As we mentioned in Remark 5.2, the qFSLP (5.1)-(5.2) can be recast as the $q$-fractional variational isoperimetric problem: Find the extremal of the functional

$$
J(y):=\int_{0}^{a}\left[p(x)\left({ }^{\mathrm{c}} D_{q, 0^{+}}^{\alpha} y\right)^{2}+r(x) y^{2}\right] d_{q} x
$$

subject to the boundary condition

$$
y(0)=y(a)=0
$$

and the isoperimetric constraint

$$
I(y)=\int_{0}^{a} w_{\alpha}(x) y^{2} d_{q} x=1
$$

The $q$-fractional Euler-Lagrange equation for the functional $I$ is

$$
2 w_{\alpha}(x) y(x)=0 \quad \text { for all } x \in A_{q, a} \text {, }
$$

which is satisfied only for the trivial solution $y=0$, because $w_{\alpha}$ is positive on $A_{q, a}$. So, no extremals for $I$ can satisfy the $q$-isoperimetric condition. If $y$ is an extremal for the $q$-fractional isoperimetric problem, then from Theorem 4.7, there exists a constant $\lambda$ such that $y$ satisfies the $q$-fractional Euler-Lagrange equation (4.9) in $A_{q, q a}^{*}$ but this is equivalent to the qFSLP (5.1). 
In the following, we shall derive a method for approximating the eigenvalues and the eigenfunctions at the same time similar to the technique in $[5,11]$. The proof follows in six steps.

Step 1. First let us point out that functional $J$ defined in (5.3) is bounded from below. Indeed, since $p, w_{\alpha}$ are positive on $A_{q, a}$,

$$
\begin{aligned}
J(y) & =\int_{0}^{a}\left[p(x)\left({ }^{\mathrm{c}} D_{q, 0^{+}}^{\alpha} y\right)^{2}+r(x) y^{2}\right] \\
& \geq \inf _{x \in A_{q, a}} \frac{r(x)}{w_{\alpha}(x)} \int_{0}^{a} w_{\alpha}(x) y^{2}(x) d_{q} x \\
& =\inf _{x \in A_{q, a}} \frac{r(x)}{w_{\alpha}(x)}=: M>-\infty .
\end{aligned}
$$

According to the Ritz method [11], p.201, we approximate a solution of (5.3)-(5.4) using the following $q$-trigonometric functions with the coefficients depending on $w_{\alpha}$ :

$$
y_{m}(x)=\frac{1}{\sqrt{w_{\alpha}}} \sum_{k=1}^{m} \frac{\beta_{k}}{\sqrt{\mu_{k}}} S_{q}\left(\frac{w_{k} x}{a}\right) .
$$

Observe that $y_{m}(0)=y_{m}(a)=0$. By substituting (5.6) into (5.3) and (5.5) we obtain

$$
\begin{aligned}
J_{m}\left(\beta_{1}, \ldots, \beta_{m}\right)= & J_{m}([\beta]) \\
= & \sum_{k, j=1}^{m} \frac{\beta_{j} \beta_{k}}{\sqrt{\mu_{j}} \sqrt{\mu_{k}}} \int_{0}^{a}\left[p(x)^{\mathrm{c}} D_{q, 0^{+}}^{\alpha} \frac{S_{q}\left(\frac{w_{k} x}{a}\right)}{\sqrt{w_{\alpha}}} D_{q, 0^{+}}^{\alpha} \frac{S_{q}\left(\frac{w_{j} x}{a}\right)}{\sqrt{w_{\alpha}}}\right. \\
& \left.+\frac{r(x)}{w_{\alpha}(x)} S_{q}\left(\frac{w_{k} x}{a}\right) S_{q}\left(\frac{w_{j} x}{a}\right)\right] d_{q} x
\end{aligned}
$$

subject to the condition

$$
I_{m}\left(\beta_{1}, \beta_{2}, \ldots, \beta_{m}\right)=I_{m}([\beta])=\frac{a \sqrt{q}}{2} \sum_{k=1}^{m} \beta_{k}^{2}=1
$$

The functions defined in (5.7) and (5.8) are functions of the $m$ variables $\beta_{1}, \beta_{2}, \ldots, \beta_{m}$. Thus, in terms of the variables $\beta_{1}, \ldots, \beta_{m}$, our problem is to minimize $J_{m}\left(\beta_{1}, \beta_{2}, \ldots, \beta_{m}\right)$ on the surface $\sigma_{m}$ of the $m$ dimensional sphere defined in (5.8). Since $\sigma_{m}$ is a compact set and $J_{m}\left(\beta_{1}, \beta_{2}, \ldots, \beta_{m}\right)$ is continuous on $\sigma_{m}, J_{m}\left(\beta_{1}, \beta_{2}, \ldots, \beta_{m}\right)$ has a minimum $\lambda_{m}^{1}$ at some point $\left(\beta_{1}^{(1)}, \ldots, \beta_{m}^{(1)}\right)$ of $\sigma_{m}$. Let

$$
y_{m}^{(1)}=\frac{1}{\sqrt{w_{\alpha}}} \sum_{k=1}^{m} \frac{\beta_{k}^{(1)}}{\mu_{k}} S_{q}\left(\frac{w_{k} x}{a}\right) .
$$

If this procedure is carried out for $m=1,2, \ldots$, we obtain a sequence of numbers $\lambda_{1}^{(1)}, \lambda_{2}^{(1)}, \ldots$, and a corresponding sequence of functions

$$
y_{1}^{(1)}(x), \quad y_{2}^{(1)}(x), \quad y_{3}^{(1)}(x), \quad \cdots
$$


Noting that $\sigma_{m}$ is the subset of $\sigma_{m+1}$ obtained by setting $\beta_{m+1}=0$, while

$$
J_{m}\left(\beta_{1}, \ldots, \beta_{m}\right)=J_{m+1}\left(\beta_{1}, \ldots, \beta_{m}, 0\right),
$$

consequently,

$$
\lambda_{m+1}^{(1)} \leq \lambda_{m}^{(1)}
$$

Increasing the domain of definition of a function can only decrease its minimum. It follows from (5.9) and the fact that $J(y)$ is bounded from below that its limit

$$
\lambda^{(1)}=\lim _{m \rightarrow \infty} \lambda_{m}^{(1)}
$$

exists.

Step 2 . We shall prove that the sequence $\left(y_{m}^{(1)}\right)_{m \in \mathbb{N}}$ contains a uniformly convergent subsequence. From now on, for simplicity, we shall write $y_{m}$ instead of $y_{m}^{(1)}$. Recall that

$$
\lambda_{m}^{(1)}=\int_{0}^{a}\left[p(x)\left({ }^{\mathrm{c}} D_{q, 0^{+}}^{\alpha} y_{m}\right)^{2}+r(x) y_{m}^{2}\right] d_{q^{x}}
$$

is convergent, so it must be bounded, i.e., there exists a constant $M_{0}>0$ such that

$$
\int_{0}^{a}\left[p(x)\left({ }^{\mathrm{c}} D_{q, 0^{+}}^{\alpha} y_{m}\right)^{2}+r(x) y_{m}^{2}\right] d_{q} x \leq M_{0}, \quad m \in \mathbb{N}
$$

Therefore, for all $m \in \mathbb{N}$ we have the inequality

$$
\begin{aligned}
\int_{0}^{a} p(x)\left({ }^{\mathrm{c}} D_{q, 0^{+}}^{\alpha} y_{m}\right)^{2} d_{q} x & \leq M_{0}+\left|\int_{0}^{a} r(x) y_{m}^{2}(x) d_{q} x\right| \\
& \leq M_{0}+\sup _{x \in A_{q, a}}\left|\frac{r(x)}{w_{\alpha}(x)}\right| \int_{0}^{a} w_{\alpha}(x) y_{m}^{2}(x) d_{q} x \\
& :=M_{0}+\sup _{x \in A_{q, a}}\left|\frac{r(x)}{w_{\alpha}(x)}\right|=: M_{1} .
\end{aligned}
$$

Moreover, since $\inf _{x \in A_{q, a}} p(x)>0$ we have

$$
\left(\inf _{x \in A_{q, a}} p(x)\right) \int_{0}^{a}\left({ }^{\mathrm{c}} D_{q, 0^{+}}^{\alpha} y_{m}\right)^{2} d_{q} x \leq \int_{0}^{a} p(x)\left({ }^{\mathrm{c}} D_{q, 0^{+}}^{\alpha} y_{m}\right)^{2} d_{q} x \leq M_{1} \text {, }
$$

and hence

$$
\int_{0}^{a}\left({ }^{\mathrm{c}} D_{q, 0^{+}}^{\alpha} y_{m}\right)^{2} d_{q} x \leq \frac{M_{1}}{\inf _{x \in A_{q, a}} p(x)}=: M_{2}^{2} .
$$

Since $y_{m}(0)=0$, from $(2.15)$ and $(5.10)$

$$
\begin{aligned}
\left\|y_{m}\right\| & =\left\|I_{q, 0^{+}}^{\alpha}{ }^{\mathrm{c}} D_{q, 0^{+}}^{\alpha} y_{m}\right\| \leq \tilde{M}_{\alpha}\left\|{ }^{\mathrm{c}} D_{q, 0^{+}}^{\alpha} y_{m}\right\|_{2} \\
& \leq \widetilde{M}_{\alpha} M_{2}
\end{aligned}
$$


for $\alpha>1 / 2$. Hence, $\left(y_{m}\right)_{m}$ is uniformly bounded on $A_{q, a}^{*}$. Now we prove that the sequence $\left(y_{m}\right)_{m}$ is equicontinuous. Let $x_{1}, x_{2} \in A_{q, a}$. Assume that $x_{1}<x_{2}$. Applying the Schwarz inequality and (2.9)

$$
\begin{aligned}
& \Gamma_{q}(\alpha)\left|y_{m}\left(x_{2}\right)-y_{m}\left(x_{1}\right)\right| \\
& =\Gamma_{q}(\alpha)\left|I_{q, 0^{+}}^{\alpha} D_{q, 0^{+}}^{\alpha} y_{m}\left(x_{2}\right)-I_{q, 0^{+}}^{\alpha} D_{q, 0^{+}}^{\alpha} y_{m}\left(x_{2}\right)\right| \\
& =\left|x_{2}^{\alpha-1} \int_{0}^{x_{2}}\left(q t / x_{2} ; q\right)_{\alpha-1}{ }^{\mathrm{c}} D_{q, 0^{+}}^{\alpha} y_{m}(t) d_{q} t-x_{1}^{\alpha-1} \int_{0}^{x_{1}}\left(q t / x_{1} ; q\right)_{\alpha-1}{ }^{\mathrm{c}} D_{q, 0^{+}}^{\alpha} y_{m}(t) d_{q} t\right| \\
& \leq\left|x_{2}^{\alpha-1} \int_{x_{1}}^{x_{2}}\left(q t / x_{2} ; q\right)_{\alpha-1}{ }^{\mathrm{c}} D_{q, 0^{+}}^{\alpha} y_{m}(t) d_{q} t\right| \\
& \quad+\left|\int_{0}^{x_{1}}\left\{x_{2}^{\alpha-1}\left(q t / x_{2} ; q\right)_{\alpha-1}-x_{2}^{\alpha-1}\left(q t / x_{1} ; q\right)_{\alpha-1}\right\}^{\mathrm{c}} D_{q, 0^{+}}^{\alpha} y_{m}(t) d_{q} t\right| \\
& \leq M_{2}\left(x_{2}^{2 \alpha-2} \int_{x_{1}}^{x_{2}}\left(q t / x_{2} ; q\right)_{\alpha-1}^{2} d_{q} t\right)^{1 / 2} \\
& \quad+M_{2}\left(\int_{0}^{x_{1}}\left(x_{2}^{\alpha-1}\left(q t / x_{2} ; q\right)_{\alpha-1}-x_{1}^{\alpha-1}\left(q t / x_{1} ; q\right)_{\alpha-1}\right)^{2} d_{q} t\right)^{1 / 2} .
\end{aligned}
$$

Since $x_{1}<x_{2}$, we have

$$
x_{2}^{\alpha-1}\left(q t / x_{2} ; q\right)_{\alpha-1} \leq x_{1}^{\alpha-1}\left(q t / x_{1} ; q\right)_{\alpha-1} \quad \text { for all } t<x_{1}<x_{2} .
$$

Using the inequality

$$
t_{1} \geq t_{2} \geq 0 \rightarrow\left(t_{1}-t_{2}\right)^{2} \leq t_{1}^{2}-t_{2}^{2}
$$

we obtain

$$
\begin{aligned}
& \int_{0}^{x_{1}}\left(x_{2}^{\alpha-1}\left(q t / x_{2} ; q\right)_{\alpha-1}-x_{1}^{\alpha-1}\left(q t / x_{1} ; q\right)_{\alpha-1}\right)^{2} d_{q} t \\
& \leq \int_{0}^{x_{1}} x_{1}^{2 \alpha-2}\left(q t / x_{1} ; q\right)_{\alpha-1}^{2} d_{q} t-\int_{0}^{x_{1}} x_{2}^{2 \alpha-2}\left(q t / x_{2} ; q\right)_{\alpha-1}^{2} d_{q} t \\
& =\int_{x_{1}}^{x_{2}} x_{2}^{2 \alpha-2}\left(q t / x_{2} ; q\right)_{\alpha-1}^{2} d_{q} t+\int_{0}^{x_{1}} x_{1}^{2 \alpha-2}\left(q t / x_{1} ; q\right)_{\alpha-1}^{2} d_{q} t \\
& \quad-\int_{0}^{x_{2}} x_{2}^{2 \alpha-2}\left(q t / x_{2} ; q\right)_{\alpha-1}^{2} d_{q} t \\
& =\int_{x_{1}}^{x_{2}} x_{2}^{2 \alpha-2}\left(q t / x_{2} ; q\right)_{\alpha-1}^{2} d_{q} t+\left(x_{1}^{2 \alpha-1}-x_{2}^{2 \alpha-1}\right) \int_{0}^{1}(q \xi ; q)_{\alpha-1}^{2} d_{q} \xi \\
& \leq \int_{x_{1}}^{x_{2}} x_{2}^{2 \alpha-2}\left(q t / x_{2} ; q\right)_{\alpha-1}^{2} d_{q} t
\end{aligned}
$$

for $\alpha>\frac{1}{2}$. Hence, we have

$$
\begin{aligned}
\left|y_{m}\left(x_{2}\right)-y_{m}\left(x_{1}\right)\right| & \leq \frac{2 M_{2}}{\Gamma_{q}(\alpha)} x_{2}^{\alpha-1}\left(\int_{x_{1}}^{x_{2}}\left(q t / x_{2} ; q\right)_{\alpha-1}^{2} d_{q} t\right)^{1 / 2} \\
& \leq \frac{2 M_{2}}{\Gamma_{q}(\alpha)\left(q^{\alpha} ; q\right)_{\infty}^{2}} x_{2}^{\alpha-1} \sqrt{x_{2}-x_{1}} \leq \frac{2 M_{2}}{\Gamma_{q}(\alpha)^{2}\left(q^{\alpha} ; q\right)_{\infty}^{2}}\left(x_{2}-x_{1}\right)^{\alpha-\frac{1}{2}} .
\end{aligned}
$$


Hence $\left\{y_{m}\right\}$ is equicontinuous. Therefore, from the Arzelà-Ascoli theorem for metric spaces, a uniformly convergent subsequence $\left(y_{m_{n}}\right)_{n \in \mathbb{N}}$ exists. It means that we can find $y^{(1)} \in C\left(A_{q, a}^{*}\right)$ such that

$$
y^{(1)}=\lim _{n \rightarrow \infty} y_{m_{n}} \text {. }
$$

Step 3. From the Lagrange multiplier at $[\beta]=\left(\beta_{1}^{(1)}, \ldots, \beta_{m}^{(1)}\right)$, we have

$$
\left.\frac{\delta}{\delta \beta_{j}}\left[J_{m}([\beta])-\lambda_{m}^{(1)} I_{m}([\beta])\right]\right|_{[\beta]=\left[\beta^{(1)}\right]}, \quad j=1,2, \ldots, m .
$$

By multiplying each equation by an arbitrary constant $c_{j}$ and summing from 1 to $m$, we obtain

$$
0=\left.\sum_{j=1}^{m} c_{j} \frac{\delta}{\delta \beta_{j}}\left[J_{m}([\beta])-\lambda_{m}^{(1)} I_{m}([\beta])\right]\right|_{[\beta]=\left[\beta^{(1)}\right]} .
$$

For $m \in \mathbb{N}$, set

$$
h_{m}(x):=\frac{1}{\sqrt{w_{\alpha}}} g_{m}(x) ; \quad g_{m}(x):=\sum_{j=1}^{m} \frac{c_{j}}{\sqrt{\mu_{j}}} S_{q}\left(\frac{w_{j} x}{a}\right)
$$

According to Proposition 3.10, we can choose the coefficients $c_{j}$ such that there exists a function $g$ satisfying

$$
\lim _{m \rightarrow \infty} D_{q}^{k} g_{m}=D_{q}^{k} g \quad(k=0,1,2)
$$

and the convergence is in $L_{q}^{2}\left(A_{q, a}^{*}\right)$ norm. Hence

$$
\lim _{m \rightarrow \infty}\left\|D_{q}^{k} h_{m}-D_{q}^{k} h\right\|_{2}=0 \quad(k=0,1,2) .
$$

We can write (5.12) in the form

$$
0=\int_{0}^{a}\left[p(x)^{\mathrm{c}} D_{q, 0^{+}}^{\alpha} y_{m}{ }^{\mathrm{c}} D_{q, 0^{+}}^{\alpha} h_{m}+\left(r(x)-\lambda_{m}^{1} w_{\alpha}(x)\right) y_{m} h_{m}\right] d_{q^{x}}
$$

Since $y_{m}(0)=0$, from $(2.11)$

$$
{ }^{\mathrm{c}} D_{q, 0^{+}}^{\alpha} y_{m}=D_{q, 0^{+}}^{\alpha} y_{m}=D_{q} I_{q, 0^{+}}^{1-\alpha} y_{m} .
$$

Then replacing ${ }^{\mathrm{c}} D_{q, 0^{+}}^{\alpha} y_{m}$ by $D_{q, 0^{+}}^{\alpha} y_{m}$ in (5.14) and applying the $q$-integration by parts rule (1.10), we obtain

$$
\begin{aligned}
0= & I_{m} \\
:= & -\int_{0}^{a} D_{q} p(x)^{\mathrm{c}} D_{q, 0^{+}}^{\alpha} h_{m}(x)\left(I_{q, 0^{+}}^{1-\alpha} y_{m}\right)(q x) d_{q} x \\
& -\int_{0}^{a} p(q x) D_{q}^{\mathrm{c}} D_{q, 0^{+}}^{\alpha} h_{m}(x)\left(I_{q, 0^{+}}^{1-\alpha} y_{m}\right)(q x) d_{q^{x}} x
\end{aligned}
$$




$$
\begin{aligned}
& +\int_{0}^{a}\left[r(x)-\lambda_{m}^{(1)} w_{\alpha}(x)\right] y_{m} h_{m} d_{q} x \\
& +\left.p(x)^{\mathrm{c}} D_{q, 0^{+}}^{\alpha} h_{m}(x) I_{q, 0^{+}}^{1-\alpha} y_{m}(x)\right|_{x=0} ^{x=a} .
\end{aligned}
$$

In the following we shall prove that

$$
\begin{aligned}
I:= & \lim _{m \rightarrow \infty} I_{m} \\
= & \int_{0}^{a}-D_{q} p(x)^{\mathrm{c}} D_{q, 0^{+}}^{\alpha} h(x)\left(I_{q, 0^{+}}^{1-\alpha} y^{(1)}\right)(q x) d_{q^{x}} \\
& -\int_{0}^{a} p(q x) D_{q}{ }^{\mathrm{c}} D_{q, 0^{+}}^{\alpha} h(x)\left(I_{q, 0^{+}}^{1-\alpha} y^{(1)}\right)(q x) d_{q} x \\
& +\left.p(x)^{\mathrm{c}} D_{q, 0^{+}}^{\alpha} h(x) I_{q, 0^{+}}^{1-\alpha} y(x)\right|_{x=0} ^{x=a}+\int_{0}^{a}\left[r(x)-\lambda^{(1)} w_{\alpha}(x)\right] y^{(1)} h d_{q} x .
\end{aligned}
$$

Indeed,

$$
\begin{aligned}
\mid I_{m}- & I \mid \\
\leq & \int_{0}^{a}\left|D_{q} p(x)\left[{ }^{\mathrm{c}} D_{q, 0^{+}}^{\alpha} h_{m}(x)\left(I_{q, 0^{+}}^{1-\alpha} y_{m}\right)(q x)-{ }^{\mathrm{c}} D_{q, 0^{+}}^{\alpha} h(x)\left(I_{q, 0^{+}}^{1-\alpha} y^{(1)}\right)(q x)\right]\right| d_{q} x \\
& +\int_{0}^{a}\left|p(q x)\left[D_{q}{ }^{\mathrm{c}} D_{q, 0^{+}}^{\alpha} h_{m}(x)\left(I_{q, 0^{+}}^{1-\alpha} y_{m}\right)(q x)-D_{q}{ }^{\mathrm{c}} D_{q, 0^{+}}^{\alpha} h(x)\left(I_{q, 0^{+}}^{1-\alpha} y^{(1)}\right)(q x)\right]\right| d_{q x} \\
& +\left|p(x)^{\mathrm{c}} D_{q, 0^{+}}^{\alpha} h_{m}(x) I_{q, 0^{+}}^{1-\alpha} y_{m}(x)-p(x)^{\mathrm{c}} D_{q, 0^{+}}^{\alpha} h(x) I_{q, 0^{+}}^{1-\alpha} y^{(1)}(x)\right|_{x=0}^{x=a} \\
& +\int_{0}^{a}\left|\left[r(x)-\lambda_{m}^{(1)} w_{\alpha}(x)\right] y_{m} h_{m}-\left[r(x)-\lambda^{(1)} w_{\alpha}(x)\right] y^{(1)} h\right| d_{q^{x}} x .
\end{aligned}
$$

For the first $q$-integral in (5.16), by adding and subtracting the term

$$
D_{q} p(x)^{\mathrm{c}} D_{q, 0^{+}}^{\alpha} h(x)\left(I_{q, 0^{+}}^{1-\alpha} y_{m}\right)(q x)
$$

to the integrand, we obtain

$$
\begin{aligned}
& \int_{0}^{a}\left|D_{q} p(x)\left[{ }^{\mathrm{c}} D_{q, 0^{+}}^{\alpha} h_{m}(x)\left(I_{q, 0^{+}}^{1-\alpha} y_{m}\right)(q x)-{ }^{\mathrm{c}} D_{q, 0^{+}}^{\alpha} h(x)\left(I_{q, 0^{+}}^{1-\alpha} y^{(1)}\right)(q x)\right]\right| d_{q} x \\
& \leq\left\|D_{q} p\right\|\left\|{ }^{\mathrm{c}} D_{q, 0^{+}} h\right\|_{\infty}\left\|\left(I_{q, 0^{+}}^{1-\alpha} y_{m}\right)(q x)-\left(I_{q, 0^{+}}^{1-\alpha} y^{(1)}\right)(q x)\right\|_{1} \\
& \quad+\left\|D_{q} p\right\| M_{3} K_{1-\alpha}\left\|{ }^{\mathrm{c}} D_{q, 0^{+}}^{\alpha}\left(h_{m}-h\right)\right\|_{2} \\
& \leq \frac{\left\|D_{q} p\right\|}{q}\left\{\left\|{ }^{\mathrm{c}} D_{q, 0^{+}} h\right\|_{\infty}\left\|I_{q, 0^{+}}^{1-\alpha} y_{m}-I_{q, 0^{+}}^{1-\alpha} y^{(1)}\right\|_{1}+M_{3} K_{1-\alpha}\left\|{ }^{\mathrm{c}} D_{q, 0^{+}}^{\alpha}\left(h_{m}-h\right)\right\|_{2}\right\},
\end{aligned}
$$

where $K_{1-\alpha}$ is the constant defined in (2.13) and $M_{3}:=\sup _{m \in \mathbb{N}}\left\|y_{m}\right\|_{\infty}$. From (5.11) and (5.13)

$$
\lim _{m \rightarrow \infty}\left\|y_{m}-y^{(1)}\right\|=\lim _{m \rightarrow \infty}\left\|D_{q} h_{m}-D_{q} h\right\|_{2}=0
$$

then applying (2.12)-(2.14), we obtain

$$
\lim _{m \rightarrow \infty}\left\|I_{q, 0^{+}}^{1-\alpha} y_{m}-I_{q, 0^{+}}^{1-\alpha} y^{(1)}\right\|_{1}=\lim _{m \rightarrow \infty}\left\|{ }^{\mathrm{c}} D_{q, 0^{+}}^{\alpha}\left(h_{m}-h\right)\right\|_{2}=0
$$


and the first $q$-integral vanishes as $m \rightarrow \infty$. As for the second $q$-integral, we add and subtract the term $p(q x) D_{q}{ }^{\mathrm{c}} D_{q, 0^{+}}^{\alpha} h(x)\left(I_{q, 0^{+}}^{1-\alpha} y_{m}\right)(q x)$. This gives

$$
\begin{aligned}
& \int_{0}^{a}\left|p(q x)\left[D_{q}{ }^{c} D_{q, 0^{+}}^{\alpha} h_{m}(x)\left(I_{q, 0^{+}}^{1-\alpha} y_{m}\right)(q x)-D_{q}{ }^{\mathrm{c}} D_{q, 0^{+}}^{\alpha} h(x)\left(I_{q, 0^{+}}^{1-\alpha} y^{(1)}\right)(q x)\right]\right| d_{q} x \\
& \leq\|p\|\left\|D_{q}{ }^{\mathrm{c}} D_{q, 0^{+}}^{\alpha} h\right\|_{2}\left\|\left(I_{q, 0^{+}}^{1-\alpha} y_{m}\right)(q x)-\left(I_{q, 0^{+}}^{1-\alpha} y^{(1)}\right)(q x)\right\|_{2} \\
& \quad+\|p\| M_{3} K_{1-\alpha}\left\|D_{q}{ }^{c} D_{q, 0^{+}}^{\alpha}\left(h_{m}-h\right)\right\|_{2} \\
& \leq \frac{\|p\|}{q}\left\{\left\|D_{q}{ }^{\mathrm{c}} D_{q, 0^{+}}^{\alpha} h\right\|_{2}\left\|I_{q, 0^{+}}^{1-\alpha} y_{m}-I_{q, 0^{+}}^{1-\alpha} y^{(1)}\right\|_{2}+M_{3} K_{1-\alpha}\left\|D_{q}{ }^{\mathrm{c}} D_{q, 0^{+}}^{\alpha}\left(h_{m}-h\right)\right\|_{2}\right\} .
\end{aligned}
$$

Since $D_{q}{ }^{\mathrm{c}} D_{q, 0^{+}}^{\alpha} f(x)=I_{q}^{1-\alpha} D_{q}^{2} f$ if $D_{q} f(0)=0$, and since $\lim _{m \rightarrow \infty}\left\|D_{q}^{2} h_{m}-D_{q}^{2} h\right\|_{2}=0$, from (2.14), the second $q$-integral tends to zero as $m$ tends to $\infty$. For the next two terms, we have for $x=0, a$,

$$
\left(I_{q, 0^{+}}^{1-\alpha} y_{m}\right)(q x)=q^{1-\alpha} I_{q, 0^{+}}^{\alpha} y_{m}(q x) \rightarrow q^{1-\alpha} I_{q, 0^{+}}^{\alpha} y^{(1)}(q x)
$$

resulting from the convergence of the sequence $\left\|y_{m}-y\right\| \rightarrow 0$, and at the points $x=0$, $x=a$, we obtain

$$
D_{q, 0^{+}}^{\alpha} h_{m}(0) \rightarrow D_{q, 0^{+}}^{\alpha} h(0), \quad D_{q, 0^{+}}^{\alpha} h_{m}(a) \rightarrow D_{q, 0^{+}}^{\alpha} h(a) .
$$

Therefore,

$$
\left|p(x) D_{q, 0^{+}}^{\alpha} h_{m}(x) I_{q, 0^{+}}^{1-\alpha} y_{m}(x)-p(x) D_{q, 0^{+}}^{\alpha} h(x) I_{q, 0^{+}}^{1-\alpha} y^{(1)}(x)\right|_{x=0}^{x=a}=0 .
$$

Similarly, the last term in the estimation (5.16) vanishes as $m \rightarrow \infty$.

Step 4 . We have

$$
I=\int_{0}^{a} p(x)^{\mathrm{c}} D_{q, 0^{+}}^{\alpha} y(x)^{\mathrm{c}} D_{q, 0^{+}}^{\alpha} h(x)+\left(r(x)-\lambda w_{\alpha}\right) y(x) h(x) d_{q} x=0 .
$$

Set

$$
\begin{aligned}
& \gamma_{1}(x):=p(x)^{\mathrm{c}} D_{q, 0^{+}}^{\alpha} y(x), \\
& \gamma_{2}(x):=\left(r(x)-\lambda w_{\alpha}\right) y(x) .
\end{aligned}
$$

Thus, since $h(0)=h(a)=0$,

$$
I=\int_{0}^{a}\left[\left(I_{q, a^{-}}^{1-\alpha} \gamma_{1}\right)(x)-\left(I_{q, 0^{+}} \gamma_{2}\right)(q x)\right] D_{q} h(x) d_{q^{\prime}} x=0 .
$$

Hence, from Lemma 4.4 there is a constant $c$ such that

$$
\left(I_{q, a^{-}}^{1-\alpha} \gamma_{1}\right)(x)-\left(I_{q, 0^{+}} \gamma_{2}\right)(q x)=c, \quad \forall x \in A_{q, a^{*}}^{*}
$$

Acting on the two sides of (5.19) by $-\frac{1}{q} D_{q^{-1}}$, we obtain

$$
D_{q, a^{-}}^{\alpha} \gamma_{1}(x)+\gamma_{2}(x)=0, \quad x \in A_{q, q a}^{*} .
$$

Hence, $y$ is a solution of the qFSLP. 
Step 5. In the following, we show that $\left(y_{m}^{(1)}\right)_{m \in \mathbb{N}}$ itself converges to $y^{(1)}$. First, from Theorem 3.12 of [34], for a given $\lambda$ the solution of

$$
\left[D_{q, a^{-}}^{\alpha} p(x)^{\mathrm{c}} D_{q, 0^{+}}^{\alpha} y+r(x)\right] y(x)=\lambda w_{\alpha}(x) y(x)
$$

subject to the boundary conditions

$$
y(0)=y(a)=0
$$

and the normalization condition

$$
\int_{0}^{a} w_{\alpha}(x) y^{2}(x) d_{q} x=1
$$

is unique except for a sign. Let us assume that $y^{(1)}$ solves (5.20) and the corresponding eigenvalue is $\lambda=\lambda^{(1)}$. Suppose that $y^{(1)}$ is nontrivial, i.e., there exists $x_{0} \in A_{q, q a}^{*}$ such that $y\left(x_{0}\right) \neq 0$ and choose the sign so that $y^{(1)}\left(x_{0}\right)>0$. Similarly, for all $m \in \mathbb{N}$, let $y_{m}^{(1)}$ solve (5.20) with corresponding eigenvalue $\lambda=\lambda_{m}^{(1)}$, and let us choose the sign so that $y_{m}^{(1)}\left(x_{0}\right) \geq 0$. Now, suppose that $\left(y_{m}^{(1)}\right)$ does not converge to $y^{(1)}$. It means that we can find another subsequence of $y_{m}^{(1)}$ such that it converges to another solution $\tilde{y}^{(1)}$. But for $\lambda=\lambda^{(1)}$, the solution of (5.20)(5.22) is unique except for a sign, hence

$$
\tilde{y}^{(1)}=-y^{(1)}
$$

and we must have $\tilde{y}^{(1)}\left(x_{0}\right)<0$. However, this is impossible because for all $m \in \mathbb{N}$, $y_{m}^{(1)}\left(x_{0}\right) \geq 0$. This is a contradiction, hence the solution is unique.

Step 6. In order to find the eigenfunction $y^{(2)}$ and the corresponding eigenvalue $\lambda^{(2)}$, we minimize the functional (5.3) subject to (5.4) and (5.5) but now with an extra orthogonality condition,

$$
\int_{0}^{a} y(x) y^{(1)}(x) w_{\alpha}(x) d_{q} x=0
$$

If we approximate the solution by

$$
y_{m}(x)=\frac{1}{\sqrt{w_{\alpha}}} \sum_{k=1}^{m} \frac{\beta_{k}}{\sqrt{\mu_{k}}} S_{q}\left(\frac{w_{k} x}{a}\right), \quad y_{m}(0)=y_{m}(a)=0,
$$

then we again obtain the quadratic form (5.7). However, admissible solutions are satisfying (5.8) together with

$$
\frac{a \sqrt{q}}{2} \sum_{k=1}^{m} \beta_{k} \beta_{k}^{(1)}=0
$$

i.e., they lie in the $(m-1)$-dimensional sphere. As before, we find that the function $\widetilde{J}([\beta])$ has a minimum $\lambda_{m}^{(2)}$ and there exists $\lambda^{(2)}$ such that

$$
\lambda^{(2)}=\lim _{m \rightarrow \infty} \lambda_{m}^{(2)}
$$


because $J(y)$ is bounded from below. Moreover, it is clear that

$$
\lambda^{(1)} \leq \lambda^{(2)}
$$

The function $y_{m}^{(2)}$ defined by

$$
y_{m}^{(2)}(x):=\frac{1}{\sqrt{w_{\alpha}}} \sum_{k=1}^{m} \frac{\beta_{k}^{(2)}}{\sqrt{\mu_{k}}} S_{q}\left(\frac{w_{k} x}{a}\right)
$$

achieves its minimum $\lambda_{m}^{(2)}$, where $\beta^{(2)}=\left(\beta_{1}^{(2)}, \ldots, \beta_{m}^{(2)}\right)$ is the point satisfying (5.8) and (5.23). By the same argument as before, we can prove that the sequence $\left(y_{m}^{(2)}\right)$ converges uniformly to a limit function $y^{(2)}$, which satisfies the qFSLP (5.1) with $\lambda^{(2)}$, boundary conditions (5.4) and orthogonality condition (5.5). Therefore, the solution $y^{(2)}$ of the qFSLP corresponding to the eigenvalue $\lambda^{(2)}$ exists. Furthermore, because orthogonal functions cannot be linearly dependent, and since only one eigenfunction corresponds to each eigenvalue (except for a constant factor) we have the strict inequality

$$
\lambda^{(1)}<\lambda^{(2)}
$$

instead of (5.24). Finally, if we repeat the above procedure with similar modifications, we can obtain the eigenvalues $\lambda^{(3)}, \lambda^{(4)}, \ldots$ and the corresponding eigenvectors $y^{(3)}, y^{(4)}, \ldots$.

\subsection{The first eigenvalue}

Definition 5.4 The Rayleigh quotient for the $q$-fractional Sturm-Liouville problem (5.1)(5.2) is defined by

$$
R(y):=\frac{J(y)}{I(y)}
$$

where $J(y)$ and $I(y)$ are given by (5.3) and (5.5), respectively.

Theorem 5.5 Let $y$ be a non-zero function satisfying $y$ and ${ }^{\mathrm{c}} D_{q, 0^{+}}^{\alpha} y$ be in $C\left(A_{q, a} *\right)$ and $y(0)=y(a)=0$. Then $y$ is a minimizer of $R(y)$ and $R(y)=\lambda$ if and only if $y$ is an eigenfunction of problem (5.1)-(5.2) associated with $\lambda$. That is, the minimum value of $R$ at $y$ is the first eigenvalue $\lambda^{(1)}$.

Proof First, we prove the necessity. Assume that $y$ is a non-zero minimizer of $R(y)$ and $R(y)=\lambda$. Consider the one parameter family of curves

$$
y=y+h \eta, \quad|h| \leq \epsilon,
$$

where $\eta$ and ${ }^{\mathrm{c}} D_{q, 0^{+}}^{\alpha}$ are $C\left(A_{q, a}^{*}\right)$ functions and $\eta(0)=\eta(a)=0$ and $\eta \neq 0$. Define functions $\phi, \psi, \xi$ on $[-\epsilon, \epsilon]$ by

$$
\phi(h):=I(y+h \eta), \quad \psi(h):=J(y+h \eta), \quad \xi(h)=R(y+h \eta)=\frac{\psi(h)}{\phi(h)}, \quad h \in[-\epsilon, \epsilon] .
$$


Hence $\xi$ is $C^{1}$ function on $[-\epsilon, \epsilon]$. Since $\xi(0)=R(y), 0$ is a minimum value of $\xi$. Consequently, $\xi_{i}^{\prime}(0)=0$. But

$$
\xi^{\prime}(h)=\frac{1}{\phi(h)}\left(\psi^{\prime}(h)-\frac{\psi(h)}{\phi(h)} \phi^{\prime}(h)\right)
$$

and

$$
\begin{aligned}
& \psi^{\prime}(0)=2 \int_{0}^{a}\left[p(x)^{\mathrm{c}} D_{q, 0^{+}}^{\alpha} y^{\mathrm{c}} D_{q, 0^{+}}^{\alpha} \eta+r(x) y \eta\right] d_{q} x, \\
& \phi^{\prime}(0)=2 \int_{0}^{a} w_{\alpha}(x) y(x) \eta(x) d_{q} x, \\
& \frac{\psi(0)}{\phi(0)}=R(y)=\lambda .
\end{aligned}
$$

Therefore,

$$
\xi^{\prime}(0)=\frac{2}{I(y)}\left(\int_{0}^{a}\left[p(x)^{\mathrm{c}} D_{q, 0^{+}}^{\alpha} y^{\mathrm{c}} D_{q, 0^{+}}^{\alpha} \eta+\left(r(x) y-\lambda w_{\alpha}\right) \eta\right] d_{q^{x}}\right) .
$$

Using (2.19), we obtain

$$
\int_{0}^{a}\left[D_{q, a^{-}}^{\alpha} p(x)^{\mathrm{c}} D_{q, 0^{+}}^{\alpha} y(x)+(r(x)-\lambda) w_{\alpha}(x) y(x)\right] \eta(x) d_{q^{\prime}} x=0 .
$$

Applying Lemma 4.3, we obtain

$$
D_{q, a^{-}}^{\alpha} p(x)^{\mathrm{c}} D_{q, 0^{+}}^{\alpha} y(x)+r(x) y(x)=\lambda w_{\alpha}(x) y(x), \quad x \in A_{q, q a}^{*}
$$

This proves the necessity. Now we prove the sufficiency. Assume that $y$ is an eigenfunction of (5.1)-(5.2) associated with an eigenvalue $\lambda$. Then

$$
D_{q, a^{-}}^{\alpha} p(x)^{\mathrm{c}} D_{q, 0^{+}}^{\alpha} y(x)+r(x) y(x)=\lambda w_{\alpha}(x) y(x), \quad x \in A_{q, q a^{*}}^{*}
$$

Multiplying (5.25) by $y$ and calculating the $q$-integration from 0 to $a$, we obtain

$$
\int_{0}^{a}\left[y(x) D_{q, a^{-}}^{\alpha} p(x)^{\mathrm{c}} D_{q, 0^{+}}^{\alpha} y(x)+r(x) y^{2}(x)\right] d_{q^{\prime}} x=\lambda \int_{0}^{a} w_{\alpha}(x) y^{2}(x) d_{q} x .
$$

Since $y \neq 0, \int_{0}^{a} w_{\alpha}(x) y^{2}(x) d_{q} x>0$ and

$$
\frac{\int_{0}^{a}\left[y(x) D_{q, a^{-}}^{\alpha} p(x)^{\mathrm{c}} D_{q, 0^{+}}^{\alpha} y(x)+r(x) y^{2}(x)\right] d_{q^{x}} x}{\int_{0}^{a} w_{\alpha}(x) y^{2}(x) d_{q^{x}} x}=\lambda .
$$

That is, $R(y)=\lambda$. Therefore, any minimum value of $J$ is an eigenvalue and it is attained at the associated eigenfunction. Therefore the minimum value of $J$ is the smallest eigenvalue. 


\section{Conclusion and future work}

This paper is the first paper that deals with variational problems of functionals defined in terms of Jackson $q$-integral on a finite domain and where the left-sided Caputo $q$-derivative appears in the integrand. We give a fractional $q$-analog of the Euler-Lagrange equation and a $q$-isoperimetric problem is defined and solved. We use these results in recasting the qFSLP under consideration as a $q$-isoperimetric problem, and then we solve it by a technique similar to the one used in solving regular Sturm-Liouville problems in [11] and fractional Sturm-Liouville problems in [5]. This completes the work started by the author in [34], and it generalizes the study of integer Sturm-Liouville problem introduced by Annaby and Mansour in [1, 2]. A similar study for the fractional Sturm-Liouville problem

$$
{ }^{\mathrm{c}} D_{q, a^{-}} p(x) D_{q, 0^{+}}^{\alpha} y(x)+r(x) y(x)=\lambda w_{\alpha}(x) y(x)
$$

is in progress.

\section{Competing interests}

The author declares that she has no competing interests.

\section{Acknowledgements}

The author thanks the referee for a number of helpful suggestions for improvement in the article. This work was funded by DSFP program of the King Saud University in Riyadh through grant DSFP/Math01.

Received: 11 March 2016 Accepted: 2 August 2016 Published online: 11 August 2016

\section{References}

1. Annaby, M, Mansour, Z: Basic Sturm-Liouville problems. J. Phys. A, Math. Gen. 38, 3775-3797 (2005)

2. Annaby, M, Mansour, Z: Corrigendum: 'Basic Sturm-Liouville problems' [J. Phys. A 38 (2005), no. 17, 3775-3797; MR2145378]. J. Phys. A, Math. Gen. 39, 8747 (2006)

3. Sturm, C, Liouville, J: Extrait d'un mémoire sur le développement des fonctions en sèries dont les différents termes sont assujettis à satisfaire à une même équation différentielle linéaire, contenant un paramètre variable. J. Math. Pures Appl. 2, 220-223 (1837)

4. Amrein, W, Hinz, A, Pearson, D (eds.): Sturm-Liouville Theory: Past and Present. Birkhäuser, Basel (2005)

5. Klimek, M, Odzijewicz, T, Malinowska, A: Variational methods for the fractional Sturm-Liouville problem. J. Math. Anal. Appl. 416, 402-426 (2014)

6. Klimek, M, Agrawal, OP: Fractional Sturm-Liouville problem. Comput. Math. Appl. 66(5), 795-812 (2013)

7. Zayernouri, M, Karniadakis, G: Fractional Sturm-Liouville eigen-problems: theory and numerical approximation. J. Comput. Phys. 252, 495-517 (2013)

8. Hajji, M, Al-Mdallal, Q, Allan, F: An efficient algorithm for solving higher-order fractional Sturm-Liouville eigenvalue problems. J. Comput. Phys. 272, 550-558 (2014)

9. Ansari, A: On finite fractional Sturm-Liouville transforms. Integral Transforms Spec. Funct. 26(1), 51-64 (2015)

10. Zayernouri, M, Ainsworth, M, Karniadakis, G: Tempered fractional Sturm-Liouville eigenproblems. SIAM J. Sci. Comput. 37(4), A1777-A1800 (2015)

11. Gelfand, I, Fomin, S: Calculus of Variations. Dover, New York (2000)

12. Zettl, A: Sturm-Liouville Theory. Mathematical Surveys and Monographs, vol. 121. Am. Math. Soc., Providence (2005)

13. Almeida, R, Malinowska, A, Torres, D: Fractional Euler-Lagrange differential equations via Caputo derivatives. In: Fractional Dynamics and Control, Part 2, pp. 109-118. Springer, New York (2012)

14. Almeida, R, Torres, D: Calculus of variations with fractional derivatives and fractional integrals. Appl. Math. Lett. 22(12), 1816-1820 (2009)

15. Almeida, R, Torres, D: Necessary and sufficient conditions for the fractional calculus of variations with Caputo derivatives. Commun. Nonlinear Sci. Numer. Simul. 16(3), 1490-1500 (2011)

16. Almeida, R, Torres, D: Fractional variational calculus for nondifferentiable functions. Comput. Math. Appl. 61(10), 3097-3104 (2011)

17. Agrawal, OP: Formulation of Euler-Lagrange equations for fractional variational problems. J. Math. Anal. Appl. 272(1), 368-379 (2002)

18. Agrawal, OP: Fractional variational calculus in terms of Riesz fractional derivatives. J. Phys. A 40(24), 6287-6303 (2007)

19. Agrawal, OP: Generalized Euler-Lagrange equations and transversality conditions for FVPs in terms of Caputo derivatives. J. Vib. Control 13(9-10), 1217-1237 (2007)

20. Agrawal, OP: Fractional variational calculus and the transversality conditions. J. Phys. A 39(33), 10375-10384 (2006)

21. Lavagno, A: Basic-deformed quantum mechanics. Rep. Math. Phys. 64(1-2), 79-91 (2009)

22. Abreu, L: Sampling theory associated with q-difference equations of the Sturm-Liouville type. J. Phys. A 38(48), 10311-10319 (2005) 
23. Annaby, M, Bustoz, J, Ismail, M: On sampling theory and basic Sturm-Liouville systems. J. Comput. Appl. Math. 206, 73-85 (2007)

24. Annaby, M, Mansour, Z, Soliman, I: q-Titchmarsh-Weyl theory: series expansion. Nagoya Math. J. 205, 67-118 (2012)

25. Nemri, A, Fitouhi, A: Polynomial expansions for solutions of wave equation in quantum calculus. Matematiche 65(1), 73-82 (2010)

26. Abreu, L: Real Paley-Wiener theorems for the Koornwinder-Swarttouw q-Hankel transform. J. Math. Anal. Appl. 334(1), 223-231 (2007)

27. Andrews, GR, Askey, R, Roy, R: Special Functions. Cambridge University Press, Cambridge (1999)

28. Gasper, G, Rahman, M: Basic Hypergeometric Series. Cambridge University Press, Cambridge (2004)

29. Jackson, FH: A generalization of the function $\Gamma(n)$ and $x^{n}$. Proc. R. Soc. Lond. 74, 64-72 (1904)

30. Koelink, K, Swarttouw, R: On the zeros of the Hahn-Exton q-Bessel function and associated q-Lommel polynomials. J. Math. Anal. Appl. 186(3), 690-710 (1994)

31. Swarttouw, RF: The Hahn-Exton q-Bessel function. PhD thesis, The Technical University of Delft (1992)

32. Jackson, FH: On q-definite integrals. Q. J. Pure Appl. Math. 41, 193-203 (1910)

33. Annaby, MH, Mansour, ZS: q-Fractional Calculus and Equations. Lecture Notes in Mathematics, vol. 2056. Springer, Berlin (2012)

34. Mansour, ZS: On fractional $q$-Sturm-Liouville problem (2016). arXiv:1602.01500 [math.CA]

35. Cardoso, J: Basic Fourier series: convergence on and outside the $q$-linear grid. J. Fourier Anal. Appl. 17(1), 96-114 (2011)

36. Agarwal, R: Certain fractional $q$-integrals and q-derivatives. Proc. Camb. Philos. Soc. 66, 365-370 (1969)

37. Rajković, P, Marinković, S, Stanković, M: A generalization of the concept of $q$-fractional integrals. Acta Math. Sin. Engl. Ser. 25(10), 1635-1646 (2009)

38. Bustoz, J, Cardoso, J: Basic analog of Fourier series on a q-linear grid. J. Approx. Theory 112, 154-157 (2001)

39. Brunt, B: The Calculus of Variations. Springer, New York (2004)

40. Fort, T: Finite Differences and Difference Equations in the Real Domain. Oxford University Press, London (1948)

41. Cadzow, J: Discrete calculus of variations. Int. J. Control 11, 393-407 (1970)

42. Logan, J: Noether's theorem and the calculus of variations. PhD thesis, The Ohio State University, USA (1970)

43. Logan, J: Generalized invariant variational problems. J. Math. Anal. Appl. 38, 174-186 (1972)

44. Logan, J: First integrals in the discrete variational calculus. Aequ. Math. 9, 210-220 (1973)

45. Logan, J: Higher dimensional problems in the discrete calculus of variations. Int. J. Control 17(1), 315-320 (1973)

46. Logan, J: Some invariance identities for discrete systems. Int. J. Control 19, 919-923 (1974)

47. Harmsen, B: The discrete calculus of variations. PhD thesis, The University of Nebraska, Lincoln (1995)

48. Harmsen, B: The discrete variational problem with right focal constraints. Panam. Math. J. 5(1), 43-61 (1995)

49. Harmsen, B: The (2,2) focal discrete variational problem. Commun. Appl. Anal. 1(2), 167-183 (1997)

50. Harmsen, B: The discrete variational problem: the vector case with right focal constraints. Panam. Math. J. 6(2), 23-37 (1996)

51. Casimiro, A, Rodrigo, C: First variation formula for discrete variational problems in two independent variables. Rev. R. Acad. Cienc. Exactas Fís. Nat., Ser. A Mat. 106(1), 111-135 (2012)

52. Cheung, W: Sharp discrete inequalities and applications to discrete variational problems. J. Comput. Appl. Math. 232(2), 176-186 (2009)

53. Colombo, L, de Diego, DM, Zuccalli, M: Higher-order discrete variational problems with constraints. J. Math. Phys. 54(9), 093507 (2013)

54. Casimiro, A, Rodrigo, C: First variation formula and conservation laws in several independent discrete variables. J. Geom. Phys. 62(1), 61-86 (2012)

55. Bangerezako, G: Variational q-calculus. J. Math. Anal. Appl. 289(2), 650-665 (2004)

56. Bangerezako, G: Variational calculus on q-nonuniform lattices. J. Math. Anal. Appl. 306(1), 161-179 (2005)

57. Malinowska, AB, Torres, DFM: The Hahn quantum variational calculus. J. Optim. Theory Appl. 147(3), 419-442 (2010)

58. Malinowska, AB, Martins, N: Generalized transversality conditions for the Hahn quantum variational calculus. Optimization 62(3), 323-344 (2013)

59. Rabei, E, Nawafleh, K, Hijjawi, R, Muslih, S, Baleanu, D: The Hamilton formalism with fractional derivatives. J. Math. Anal. Appl. 327(2), 891-897 (2007)

60. Rabei, E, Ababneh, B: Hamilton-Jacobi fractional mechanics. J. Math. Anal. Appl. 344(2), 799-805 (2008)

61. El-Nabulsi, RA: Universal fractional Euler-Lagrange equation from a generalized fractional derivate operator. Cent. Eur. J. Phys. 9(1), 250-256 (2010)

62. Malinowska, A, Torres, D: The Hahn quantum variational calculus. J. Optim. Theory Appl. 147(3), 419-442 (2010)

63. Lang, S: Calculus of Several Variables, 3rd edn. Undergraduate Texts in Mathematics. Springer, New York (1987)

64. Rudin, W: Principles of Mathematical Analysis, 3rd edn. McGraw-Hill, New York (1964) 